\title{
Zur Symbolik der sogenannten Schulterrosette bei Löwendarstellungen ${ }^{1}$
}

\author{
Hierzu Tafeln XVIII-XXII
}

Die „Schulterrosetten“ der Löwen waren bereits vor geraumer Zeit Gegenstand einer wissenschaftlichen Diskussion. Den Auslöser bildete ein Artikel von H. J. Kantor ${ }^{2}$ über Entwicklung, Herkunft und Bedeutung dieser „Rosetten“ in den Löwendarstel-

\footnotetext{
Angeregt wurde der vorliegende Beitrag durch einen Vortrag des Altorientalisten R. Czichon, den ich vor einiger Zeit an der Universität Hamburg hören konnte. Czichon referierte in diesem Vortrag über „Gestaltungsprinzipien der neuassyrischen Flachbildkunst und ihre Entwicklung vom 9. bis zum 7. Jhdt. v. Chr." und wies u. a. überzeugend nach, daß die „Schulterrossetten“ der Löwen in den von ihm präsentierten Darstellungen die Stärke und Vitalität der Tiere zum Ausdruck bringen. Für Hinweise und Anregungen bei der Fertigstellung des vorliegenden Beitrages danke ich besonders P. Baum-vom Felde, M. Herb und B. Schad.
}

lungen in der bildlichen Kunst des vorderen Orients. $\mathrm{Zu}$ diesem Beitrag haben in erster Linie J. Arkell, D. Bate und N. van Buren ${ }^{5}$ kritisch Stellung bezogen.

Von naturwissenschaftlicher Seite wurde von Bate und unterstützend von Arkell dargelegt, daß diese "Rosette“ entgegen der von Kantor vertretenen These nicht ausschließlich ornamentalen Charakter besitze, sondern vielmehr eine präzise Detailwieder-

${ }^{2}$ H. J. Kantor, The „Shoulder Ornament" of Near Eastern Lions, in: JNES 6, 1947, 250-274.

J. Arkell, The Shoulder Ornament of Near Eastern Lions, in: JNES 7, 1948, 52.

${ }^{4}$ D. Bate, The "Shoulder Ornament" of Near Eastern Lions, in: JNES 9, 1950, 53-54. Erstmals ging vermutlich J. Sainte Fare Garnot, Le lion dans l'art Égyptien, in: BIFAO 37, 1937/38, 82, von einer Naturbeobachtung des Künstlers aus.

N. van Buren, An Additional Note on the Hair Whirl, in: JNES 9, 1950, 54-55. 
gabe des Künstlers, resultierend aus Naturbeobachtungen, darstelle, da festzustellen ist, daß das Fell junger, vorwiegend männlicher Löwen naturgegeben an den Schultergelenken Haarwirbel aufweist, die erst im Laufe des Wachstums des Tieres verschwinden ${ }^{6}$. Die Angabe der Haarwirbel sei in den bildlichen Wiedergaben demnach als Ausdruck der Jugend und Vitalität des männlichen Tieres zu interpretieren. Der Kritik Bates an Kantor, theoretische Überlegungen $\mathrm{zu}$ Tierdarstellungen angestellt, ohne dabei den Bezug zur Wirklichkeit untersucht zu haben, wurde u. a. entgegengehalten, daß die „Rosetten“ meistens nur stilisiert wiedergegeben seien und daher keiner natürlichen Erscheinung entsprächen ${ }^{7}$. Eine symbolische Bedeutung der „Rosette“ lehnte Kantor weitestgehend ab.

Priorität genoß in den Beiträgen Kantors und in der sich anschließenden Diskussion die Frage nach dem Ursprungsland, von dem aus die Verbreitung des Motivs der „Rosette“ den Ausgang nahm. Während Kantor Argumente für Ägypten als Herkunftsgebiet anführte, sprachen sich andere für einen vorderasiatischen Ursprung aus ${ }^{8}$.

Pongracz wertete einige Jahre später die „Rosetten" als fremdländisches Element in der ägyptischen Kunst und interpretierte insbesondere ihr Auftreten in Kombination mit den Schmuckbändern am Löwenleib als Hinweis darauf, daß eine unägyp-

\footnotetext{
${ }^{6}$ Siehe zur Veranschaulichung die Phototafel im zitierten Artikel von Bate (Taf. 2 infra p. 54), die einen jungen Zoo-Löwen mit dem Haarwirbel an der Schulter zeigt. Die Wirbel lassen sich an männlichen Jungtieren besser nachweisen als an weiblichen. Die Haarwirbel tauchen bei Löwen im Alter von etwa 2 Monaten auf und bleiben bis ca. zum dritten Lebensjahr sichtbar. Bei weiblichen Jungtieren sind sie schwächer ausgeprägt als bei den männlichen (Bate, op. cit., 53; Arkell, op. cit.). Die Haarwirbel sind außer bei Löwen- auch bei Stierdarstellungen festzustellen. Das gefleckte Fell kann ebenso auf ein Jungtier hinweisen (cf. D. Kleinsgütl, Feliden in Altägypten, Wien 1997, 29 ff.). Jedoch finden sich insbesondere im Raum Ostafrika auch Beispiele dafür, daß diese Flecken nicht verschwinden und bis ins Alter der Tiere sichtbar bleiben. Cf. dazu R. Despard Estes, The Behaviour Guide to African Mammals, Berkeley und Los Angeles 1991, 369 (Literaturhinweis M. Herb).

H. J. Kantor, A Further Comment on the Shoulder Ornament, in: JNES 9, 1950, $55 \mathrm{f}$.

${ }^{8}$ Kantor, op. cit. (n. 7), (pro Ägypten); van Buren, op. cit., (pro Mesopotamien); A. Vollgraff-Roes, The Lion with the Body Markings in Oriental Art, in: JNES 12, 1953, 40-49 (pro Mesopotamien).
}

tische Provenienz zum Ausdruck gebracht werden sollte'.

Nach der Materialsammlung von Kantor ist die „Schulterrosette" zuerst in Altägypten bezeugt und floß von dort aus in das Bilderrepertoire der Kulturen des vorderen Orients ein ${ }^{10}$. Belegen läßt sich diese Rosette - oder besser der Schulterhaarwirbel in Ägypten sowohl am Schultergelenk der Löwen als auch an der Schulter oder dem Stirnhaar von Stieren $^{11}$. Abgesehen von vereinzelten Belegen aus dem Alten und Mittleren Reich, stammt die überwiegende Zahl der Zeugnisse aus der Zeit des Neuen Reiches und der Spätzeit. Die frühesten komplett erhaltenen Darstellungen fanden sich in Denkmälern der Gemahlinnen des Pepi II. aus der 6. Dynastie in Saqqa$\mathrm{ra}^{12}$.

In Ägypten sind die Wirbel bei Löwendarstellungen des öfteren in Totenbuchvignetten nachweisbar. Hier sind vor allem die sogenannten Horizontlöwen, die in der Vignette zum 17. Kapitel des Totenbuches zu sehen sind, und die "Fresserin" in der Totengerichtsszene zu nennen. Auch in den Darstellungen der auf einem Löwen stehenden, in Syrien/Palästina beheimateten Göttin Qadesch, bei Löwen, die als Wächter fungieren, bei der Darstellung des Löwen als Wüstentier oder bei den Löwen, die zum Bildprogramm des Königsthrones gehören, sind die Haarwirbel im Fell dargestellt.

Kantor berücksichtigte bei ihrer Auswertung der ägyptischen Zeugnisse in der Regel die Funktion oder (theologische) Intention des Bildträgers nicht. Ihre Zusammenstellung der Quellen liefert einen ersten Überblick; aber es wurde versäumt, die Naturbeobachtung mit der spezifischen Funktion der Objekte und ihrer Aussage in Zusammenhang zu

M. Pongracz, Löwendarstellungen an Podesten der Königsthrone, in: MDAIK 15, 1957, 217 ff. Als Hauptargumente für die fremden Elemente nannte die Autorin die mit Rosetten und Bändern verzierten Löwen am Thronpodest und bei den Darstellungen des Löwen, auf dem Qadesch steht, sowie die heraldische, von vegetabilen Symbolen unterbrochene Reihung der Löwen.

${ }^{10}$ H. J. Kantor, The "Shoulder Ornament" of Near Eastern Lions, in: JNES 6, 1947, 250-274.

${ }^{11}$ Siehe für den Wirbel im Stirnhaar der Stiere z. B. Ostrakon Kairo 2069 (ODeM 2001-3053, Taf. XI) oder TT 336 (Nfr-rnpt): B. Bruyère, Fouilles de Deir el Médineh (1924-25), FIFAO 3, 3, Le Caire 1926, 101, Fig. 69.

${ }^{12}$ Pongracz, op. cit., 214, gibt als frühesten Beleg für das Motiv des Löwenpaares am Thron eine Darstellung im Totentempel des Sahure an. Bei diesem sind allerdings die Körper der Tiere an den entscheidenden Stellen zerstört. 
bringen. Es hat sich gezeigt, daß allein eine Belegsammlung und Erstellung einer chronologischen oder typologischen Reihe ohne Betrachtung des Kontextes, in den jedes einzelne Dokument eingebunden ist, dem Material nicht gerecht wird. Erste Ansätze zur Interpretation der Motive der mit Rosetten und Bändern geschmückten Löwen am Königsthron liegen vor $^{13}$, aber bei den übrigen Quellen steht dies noch aus.

Bis heute werden die "Schulterrosetten" in den unterschiedlichsten Kontexten weiterhin meist nur als schmückendes Element in der bildlichen Kunst zitiert; die kritischen Stellungnahmen von Arkell und Bate blieben weitgehend unberücksichtigt und wurden noch nicht in bezug auf die Tierdarstellungen in der altägyptischen Kunst angewendet ${ }^{14}$. Zwar zog in letzter Zeit beispielsweise Wilkinson ${ }^{15}$ die „Schulterrosette" in einer Arbeit über Darstellungen von Sternbildern heran, aber er identifizierte die „Rosette " in der Schulterposition des Löwen als den Stern Regulus, den hellsten Stern im Sternbild des Löwen. Diese Identifikation wurde kürzlich von Satzinger ${ }^{16}$ in Zweifel gezogen, jedoch sprach auch Satzinger nach wie vor von der "Rosette“ im Schulterfell der

${ }^{13}$ Pongracz, op. cit., $213 \mathrm{ff}$.

${ }^{14}$ Auch außerhalb der Ägyptologie scheinen die Anregungen Bates keine breite Zustimmung gefunden zu haben. Cf. S. Michel, Haarwirbel und Haarbüschel im skythischen und thrako-getischen Bilderrepertoire, in: Hamburger Beiträge zur Archäologie 18, 1991, 181 -215 (für den Literaturhinweis danke ich U. Effland). Die Autorin äußert sich skeptisch zu dem Beitrag von van Buren (Arkell und Bate sind nicht erwähnt) und vertritt in Anlehnung an Kantor die Meinung: „Auch treten Haarwirbel in der Natur nicht immer auf und sind keineswegs eine so auffällige Erscheinung, als daß man ihnen derartige Aufmerksamkeit geschenkt haben dürfte" (Michel, op. cit., 184, cf. dazu Kantor, op. cit., 253 und Arkell, op. cit., 52: „In nature such tufts are not always presented, nor are they features of such prominence as to claim attention.").

${ }^{15}$ R. H. Wilkinson, A Possible Origin for the "Shoulder Ornaments" in Egyptian Representations of Lions, in: VA 5, 1989, 59-69. Der Autor zitiert zwar den Beitrag von J. Sainte Fare Garnot, Le lion dans l'art Égyptien, in: BIFAO 37, 1937/38, 82, negiert aber - in Unkenntnis der Beiträge von Arkell und Bate (?) - die dort gegebenen Anregungen.

${ }^{16}$ H. Satzinger, The Scarab on the Cat's forehead, in: Essays in honour of Prof. Dr. Jadwiga Lipińska, Warsaw Egyptological Studies I, Warschau 1997, 399-407 und spez. 404-405: „Both the lion and the bull are animals with solar connotations in Egyptian symbolism and this may have invited the Egyptian artist (or may we say art theoreticians?) to apply the rosette ornament to them".
Löwen und im Stirnhaar der Stiere, und er führte, ausgehend von den Fellzeichnungen am Kopf von Katzen, diese „Rosetten“ auf einen gemeinsamen solaren Aspekt der Tiere zurück.

Daß die sogenannte „Rosette“ im Schulterfell des Löwen in der altägyptischen Kunst keineswegs nur als schmückendes Ornament zu werten ist, sondern vielmehr ein fundiertes zoologisches Wissen des Künstlers bezeugt, und daß diesem ikonographischen Detail darüber hinaus eine symbolische Bedeutung zukommt, die dem aufmerksamen Betrachter eine Information vermitteln kann, will ich im folgenden, ausgehend von den Beobachtungen Bates, an verschiedenen Objekten exemplarisch demonstrieren. Ich klassifiziere die Dokumente dabei in:

- die Darstellungen der Horizontlöwen im Totenbuch (\$ 1 ),

- die Szenen, die in Zusammenhang mit der königlichen Regeneration und Stärke stehen - das sind vor allem die Bilder des Königs bei der Löwenund Vogeljagd, die Löwen am Thronsitz und an der Kopfstütze (\$ 2),

- die Darstellungen der Totenfresserin im Totenbuchspruch 125 (\$ 3 ) und

- die Bilder der auf dem Löwen stehenden Göttin Qadesch (\$ 4).

\section{$\$ 1$ Die Schulterhaarwirbel in den Darstellungen der „Horizontlöwen“}

$\mathrm{Zu}$ den interessantesten Beispielen der bildlichen Wiedergabe von Haarwirbeln am Schulterfell der Löwen zählen die Haarbüschel an den Schultergelenken der sogenannten Horizontlöwen, die seit dem Neuen Reich vorwiegend in Totenbuchvignetten mehrfach bezeugt sind ${ }^{17}$.

Die Illustration zum Totenbuchspruch 17 zeigt ein antithetisch hockendes Löwenpaar, auf dessen Rücken die Sonnenscheibe im Horizontgebirge liegt ${ }^{18}$. Von dieser Horizonthieroglyphe kann ein

${ }^{17}$ Die Wiedergabe der O-Hieroglyphe mit „Horizont" ist Konvention. Nach K. Jansen-Winkeln, Horizont und Verklärtheit: Zur Bedeutung der Wurzel $3 h$, in: SAK 23, 1996, $201 \mathrm{ff}$., bezeichnet $3 h t$ präziser den zwischen himmlischer und irdischer Sphäre gelegenen Ort, an dem die Sonne wirksam ist.

${ }_{18}$ Zur Deutung des Bildes cf. zuletzt O. Keel und S. Schroer, Darstellungen des Sonnenlaufs und Toten- 
Lebenszeichen herabhängen, unter dem Löwenpaar kann in Hieroglyphen $n h h$ und über ihm die Himmelshieroglyphe stehen. Statt der Horizonthieroglyphe sieht man auch den widderköpfigen oder den falkengestaltigen Sonnengott in der Sonnenscheibe. Seltener ist das Sonnenkind in dieser Scheibe oder in einer Uroboros-Schlange dargestellt und wird dabei getragen von der Großen-Flut, der Himmelskuh Mhtwrt. Hier und da ist der Sonnengott als Skarabäus in der Scheibe, die aus dem Gebirge emporsteigt, abgebildet.

Das Löwenpaar, das die Horizonthieroglyphe trägt, wurde als Ruti bzw. als das Geschwisterpaar Schu und Tefnut oder als Erdgott Aker gedeutet ${ }^{19}$. Im übertragenen Sinne können die Löwen Re und Osiris, den Tag und die Nacht oder die Zeitbegriffe $n h h$ und $\underline{d} t$ symbolisieren. In einem Papyrus aus der 21. Dyn. verkörpern die Raubtiere das Ost- und das Westgebirge $^{20}$.

buchvignetten auf Skarabäen, in: ZÄS 125, 1998, 13-29 und zuvor: E. Hornung, Die Tragweite der Bilder, in: ERANOS Jahrbuch 48, 1979, $183 \mathrm{ff}$. (mit Diskussion der Thesen Sethes und Schäfers); W. Westendorf, Altägyptische Darstellungen des Sonnenlaufes auf der abschüssigen Himmelsbahn, MÄS 10, Berlin 1966, passim und spez. Abb. 10-12; ders., Die geteilte Himmelsgöttin, in: I. Gamer-Wallert und W. Helck (Hrsgg.), Festschrift für E. Brunner-Traut, Tübingen 1992, 341 ff.; S. Schott, Zum Weltbild der Jenseitsführer des neuen Reiches, in: NAWG Göttingen 1965, 185-197 (insbesondere zur Beziehung zwischen Ruti und Aker) sowie H. Schäfer, Weltgebäude der Alten Ägypter, Berlin und Leipzig 1928, 83-128 und K. Sethe, Altägyptische Vorstellungen vom Lauf der Sonne, SPAW Göttingen 1928, 259-284 (zur Frage, ob die Horizonthieroglyphe den Aufgang der Sonne oder deren Auf- und Untergang ins Bild setzt). Ferner zum Motiv der Horizontlöwen im Grab M. Saleh, Das Totenbuch in den thebanischen Beamtengräbern des Neuen Reiches, AV 46, Mainz 1984, 18. Für eine Darstellung im Königsgrab siehe die leider teilzerstörte Darstellung der Horizontlöwen im Grab der Nefertari (QV 66): Chr. Leblanc, Ta-set-neferou, une necropole de Thebes-ouest et son histoire I, Le Caire 1989, Taf. CLVII (nur der rechte Löwe, der deutlich einen Haarwirbel besitzt, ist erhalten).

${ }^{19}$ Cf. U. Köhler, in: LÄ III, 1082, s. v. „Löwe“; C. de Wit, Le rôle et le sens du lion dans l'Égypte ancienne, Luxor o. J. , 324-332 (im Anschluß abgekürzt mit De Wit, Le rôle et le sens du lion), 123-147 (zu Ruti als Bezeichnung des Löwenpaares); 107-122 (zu Schu und Tefnut als Löwenpaar); 158-157 (zu den Löwen und den beiden Horizonten).

${ }^{20}$ pLouvre 3292. Siehe die Abbildung bei M. G. Nagel, Un papyrus funéraire de la fin du nouvel empire, in: BIFAO 29, 1929, Taf. IV und p. 90. Der linke Löwe trägt
Bei genauerer Betrachtung der Löwen lassen sich Beispiele dafür finden, daß entweder keins der Tie$\mathrm{re}^{21}$, alle beide ${ }^{22}$ oder nur eins den Haarwirbel aufweist. Aufschlußreich ist der letzte Fall mit der Angabe nur eines Wirbels. Hierfür sind mir Beispiele sowohl in der Grabdekoration als auch auf anderen funerären Objekten wie auf Papyri und Särgen bekannt.

Als erstes Beispiel sei die für die vorliegende Fragestellung wichtigste Darstellung des Löwenpaares im Totenbuch des $3 n j$ genannt (Tafel XVIII) ${ }^{23}$. In diesem Totenbuchpapyrus, der in die späte 19. Dynastie datiert ist, hat der altägyptische Künstler dem linken der beiden Raubtiere einen Haarwirbel auf das Schultergelenk gemalt, während das rechte Äquivalent ohne dieses Attribut zu sehen ist. $\mathrm{Da}$ dies keineswegs nur zufällig ist, macht die Beischrift zur Vignette deutlich, die zusätzliche Informationen für

die Beischrift $b 3 h w$ und der rechte die Beischrift $m 3 n w$. Der dazugehörige Text erwähnt Re als den Löwen des Abends ( $r w n m^{r} r w$ ) und als das Kind am Morgen ( $h j m$ dw3jt). Zu Re als dem Löwen der Nacht cf. oCG 25207 (A. Erman, in: ZÄS 38, 1900, 23) und zum gealterten Sonnengott als dem Löwen der Unterwelt die Stele MFA 22.402 (R. J. Leprohon, CAA Boston III, Mainz 1991, 137-138): Atum ist der $r w$ st $n \quad d w 3 t$. Cf. ferner C. E. Sander-Hansen, Die Texte der Metternichstele, AnAe 7, Kopenhagen 1956, 84: Der Sonnengott ist der Stier des Ostgebirges und der Löwe ( $r w)$ des Westgebirges; Urk. II, 63,9 (= Statue CG 70031): Schu und Tefnut sind die Löwen $(m 3 j)$ des Nordens und des Südens. Zusammen werden sie Ruti genannt, als deren Vater Re oder (der gealterte Sonnengott) Atum gilt.

pHunefer (pBM 9901), siehe E. A. W. Budge, The Book of the Dead, Facsimiles of the Papyri of Hunefer, Anhai, Kerasher und Netchemet with Supplementary Text from the Papyrus of $\mathrm{Nu}$, London 1899, Taf. 8; E. A. W. Budge, The Greenfield Papyrus in the British Museum, The Funerary Papyrus of Princess Nesitanebtashru, Daughter of Painetchem II and Nesi-Khensu, and Priestess of Amen-Ra at Thebes, about B.C. 970, London 1912, Taf. LXXXII; TT 359: Jn-hr-hcw (B. Bruyère, Rapport sur les fouilles de Deir el Médineh 1930, FIFAO 8,3, Le Caire 1933, Taf. XIV) oder Tb (Naville), Taf. 27 (B. b.).

Siehe TT 360, PM I I $^{2}, 1,425$ (14)-(15) = Bruyère, op. cit., Taf. XXIX; Sarg CG 2002; pGreenfield, Taf. XLI (cf. aber daneben Taf. IX); Tb (Naville), Taf. XVII (D.a., L.a. und A.p.); cf. ferner die unten besprochene Kopfstütze Tutanchamuns (\$2).

${ }^{23}$ pBM 10470. Tafel nach der Faksimile-Ausgabe von E. Dondelinger, Papyrus Ani, Codices Selecti LXII, Graz 1978, Taf. 7; cf. R. O. Faulkner u. a., The Egyptian Book of the Dead, The Book of Going Forth by Day being the Papyrus of Ani, New York 1994. 
das Gesamtverständnis des Bildes liefert. Unter der Prämisse, daß der Haarwirbel das Kennzeichen des Jungtieres ist, ist in dieser Vignette allein optisch der linke Löwe als das Jungtier zu identifizieren. Findet bereits über die Angabe des Wirbels eine Differenzierung der beiden Löwen statt, wird die These, daß das linke Tier durch eben diesen Wirbel als Jungtier gekennzeichnet werden sollte, durch die Beischrift abgesichert. Über dem rechten Löwen steht in Hieroglyphen „das Gestern“ (sf) und über seinem Komplement „das Heute“ bzw. „das Morgen“ (dw3w). Der dazugehörige Text bezeichnet das Löwenpaar ebenfalls als „das Gestern" und „das Morgen“" und erläutert in der entsprechenden berühmten Glosse, die heute als „Quintessenz" des Totenbuches ${ }^{24}$ bezeichnet wird:

„Ich bin das Gestern. Ich kenne das Morgen. Was bedeutet das? Was das Gestern betrifft - Osiris ist es. Was das Morgen betrifft - Re ist es. ${ }^{\text {c }}{ }^{25}$

Der rechte Löwe stellt demzufolge Osiris (bzw. den gealterten Sonnengott) und der linke den jungen, regenerierten Sonnengott dar. Dadurch erklärt sich, warum im Idealfall nur einer der beiden Löwen den Haarwirbel als Kennzeichen der Jugend und Vitalität erhalten kann ${ }^{26}$. Das Beispiel im Papyrus des 3nj macht ferner deutlich, daß Text und Bild nicht isoliert betrachtet werden dürfen, sondern sich wechselseitig komplementieren und nur zusammen ein einheitliches Ganzes ergeben ${ }^{27}$.

${ }^{24}$ Siehe E. Hornung, Das Totenbuch der Ägypter, Zürich und München 1990, 424.

${ }^{25}$ Siehe Urk. V, 12,2 und als Sargtextvorläufer CT IV, p. 192-3 (Spruch 335). Cf. ferner die Bearbeitung des Spruches von U. Rößler-Köhler, Kapitel 17 des Ägyptischen Totenbuches, GOF 10, Wiesbaden 1979, 214 und 240 (Übersetzung und Auflistung der Parallelen).

${ }^{26}$ Die oben zitierten Ausnahmen sollen nicht verschwiegen werden. Aber im vorliegenden Beitrag konnten nicht alle Belege erfaßt und in ihrem spezifischen Zusammenhang interpretiert werden. Es geht mir lediglich darum, auf dieses Phänomen aufmerksam zu machen und nachzuweisen, daß mit der Angabe der Wirbel in bestimmten Fällen eine Absicht verbunden sein kann.

${ }^{27}$ Die Horizontlöwen im pAni wurden bereits u. a. von Kantor für ihre Argumentation herangezogen. Allerdings betrachtete sie den Löwen isoliert und ließ sein Äquivalent, das ohne Wirbel dargestellt ist, sowie das Umfeld und die Beischrift bei ihren Überlegungen außer acht. - Zur Ambivalenz von Text und Bild cf. J. Baines, Fecundity Figures, Egyptian Personification and the Iconology of a Genre, Warminster 1985, passim und spez. $277 \mathrm{ff}$. (zum
Bezieht man die angrenzenden Szenen mit in die Überlegungen ein, rundet sich das Bild zusätzlich ab. Vor dem linken Löwen, der den verjüngten Sonnengott symbolisiert, sieht man einen kleinen Opferaltar mit zwei Lotosblüten. Die eine der beiden Blüten öffnet sich zur Nase des Löwen hin, während die zweite zu dem Verstorbenen und seiner Frau hinzeigt, die in Gestalt von zwei b3-Vögeln auf der Grabfassade hocken. Der Lotos spendet Leben und gilt als Metapher für die Wiedergeburt. Der Verstorbene und der verjüngte Gott können gleichermaßen den Lebensodem einatmen.

Neben dem rechten Löwen, also dem gealterten Sonnengott oder Osiris, sieht man hingegen den $b n w$-Vogel (Phönix) und die Mumie des Verstorbenen, die auf der Löwenbahre liegt, von Anubis versorgt und von Isis und Nephthys in Gestalt von Geierweibchen betrauert wird. Die Darstellung des Phönix kann primär als Euphemismus für den Tod und sekundär als Symbol für die Verjüngung und Wiedergeburt aufgefaßt werden ${ }^{28}$. Nach der Balsamierung wird Osiris bzw. der Verstorbene zu einem $b n w$-Vogel. Seine zyklische Transformation wird durch die Horizonthieroglyphe, die über den Löwen schwebt, ins Bild gesetzt. Am Ende des Umwandlungsprozesses hockt er als $b 3$-Vogel vor dem jungen Löwen. Die Blickrichtung des Phönix zur Totenbahre hin sowie seine Plazierung neben den ,alten“ Löwen - das ist das Gestern, welches Osiris ist und damit der Tod, aber auch die Wiederbelebung fügen sich in die eben gemachten Beobachtungen ${ }^{29}$.

Begriff „Dekorum“) und E. Hornung, Die Tragweite der Bilder (n. 18), 214: „.. . Bilder sind offen und weisen über sich hinaus auf weitere Seinsbereiche, in ihnen ist die ganze Wirklichkeit enthalten, in ihrer Widersprüchlichkeit und Vielschichtigkeit. Wer auf die Sprache der Bilder verzichtet und in ihr eine längst überwundene Form des Ausdrucks sieht, der entfernt sich von der Wirklichkeit".

${ }^{28}$ Siehe L. Kákosy, in: LÄ IV, 1032 ff., s.v. „Phönix“ und Chr. Leitz, Tagewählerei. Das Buch h3t nhh ph.wy $d t$ und verwandte Texte, 2 Bde., ÄA 55, Wiesbaden 1994, 161-164 (zur Verwandlung des Osiris in einen bnw-Vogel am IV. 3ht 12, dem ersten Tag der Osirismysterien).

${ }^{29}$ W. Westendorf, Altägyptische Darstellungen des Sonnenlaufes auf der abschüssigen Himmelsbahn, MÄS 10, Berlin 1966, $18 \mathrm{f}$. und $83 \mathrm{f}$, identifiziert im Zusammenhang mit seinen Vorstellungen zum schrägen Himmelsbogen den höher gelegenen Löwen, das ist in diesem Fall der Löwe mit der Beischrift $s f$, anders als hier vorgeschlagen, nicht als den West-, sondern als den Osthorizont. Nach Westendorf verläuft die Neigung des Himmels vom Osten zum Westen bzw. vom Gestern zum Morgen, was zur Folge hat, daß Osiris dem Osthorizont zugeordnet ist. Zur sich 


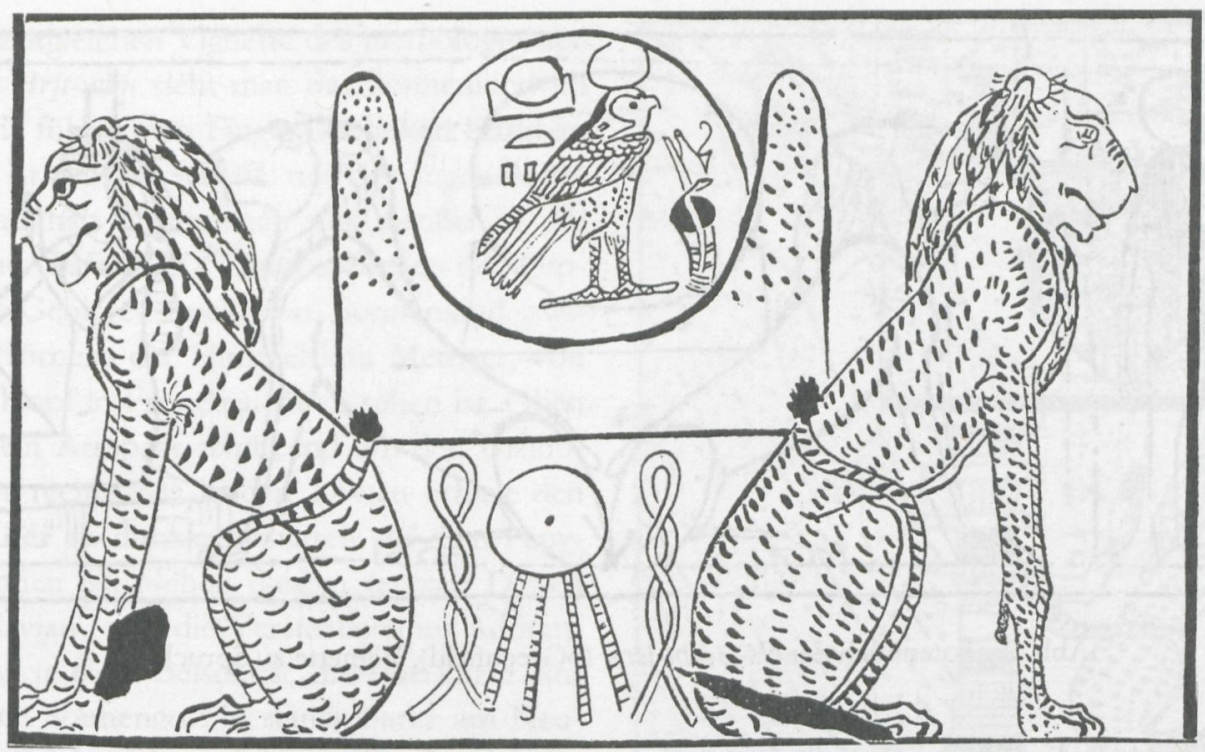

Abb. 1. Horizontlöwen im Grab des Nfr- $3 b t$ (TT 5)

Findet sich die Vermutung, daß die Angabe der Schulterhaarwirbel die Jugend des Trägers kenntlich macht, durch Bild, Bildzusammenhang und Beischriften einmal bestätigt, sollen als nächstes weitere Darstellungen von Löwen mit Blick auf die mögliche Intention, die mit der Angabe der Wirbel verbunden sein kann, betrachtet werden.

Nach der Amarnazeit wurden die Totenbuchvignetten in die Grabdekoration integriert. In einer Wandmalerei im thebanischen Grab des Nfr- $3 b t$ (TT 5) befindet sich eine Darstellung der beiden Löwen mit der Horizonthieroglyphe auf ihrem Rükken (Abb. 1) ${ }^{30}$. In der Sonnenscheibe ist der durch eine Beischrift ausgewiesene falkengestaltige ReHarachte abgebildet, und unter dem Horizontgebirge steht in Hieroglyphen $n h h$. Das linke der beiden Tiere zeigt deutlich das Haarbüschel, das nicht stilisiert als Rosette wiedergegeben und von einem Kreis umschlossen ist, sondern naturalistisch anmutet. Der rechte Löwe hingegen ist ohne dieses Dekorationselement zu sehen. Das Bildprogramm der Wand liefert auch in diesem Fall weitere Informationen

daraus ergebenden Problematik verweist Westendorf auf Totenbuchspruch 3, der besagt, daß Re im Gestern geboren wurde, und ferner auf den vergleichbaren Bedeutungswechsel bei Morgen- und Abendbarke sowie auf die Vertauschung der beiden Horizontberge $b 3 h w$ und $m 3 n w$.

${ }^{30} \mathrm{PMI}^{2}, 1$, p. 13 (14). Abb. nach J. Vandier, La Tombe de Nefer Abou, MIFAO 69, Le Caire 1935, Frontispice und cf. Taf. XVI. über das Gesamtverständnis ${ }^{31}$. Links an dieses Bild schließt sich eine Szene an, in welcher der Grabinhaber auf einer Sed-Fest-Hieroglyphe sitzend durch Horus und Thot rituell gereinigt wird. Der linke Löwe und der Verstorbene sind dabei in gleicher Augenhöhe dargestellt und blicken sich an. Im Register darüber ist die Baumgöttin zu sehen, die den Verstorbenen vor dem Grab speist, auf dem wiederum Re-Harachte in Falkengestalt hockt. Rechts daneben steht Nut im njnj-Gestus vor dem mumiengestaltigen Osiris-Chontamenti, der im Herrscherornat auf einer Grabfassade steht.

Zentrale Themen der Grabwand sind demnach Tod und Auferstehung des Verstorbenen, sein Werden zu Osiris und seine Einbindung in den Kreislauf der Regeneration. Die beiden Löwen mit der Horizonthieroglyphe in ihrer Mitte symbolisieren wie im $\mathrm{Pa}$ pyrus des $3 n j$ diesen Kreislauf. Vergleichbar dem ägyptischen Tempel stellen sie als Tor zur Unterwelt die Nahtstelle zwischen diesseitiger und jenseitiger Welt dar ${ }^{32}$. Im Horizont findet der Verstorbene ,den Einstieg in die Welt der Toten", und der Sonnengott geht gleichsam durch dieses "Weltentor" auf und

${ }^{31}$ Siehe Vandier, op. cit., Taf. XVI-XVIII.

Cf. zum Tempel als Horizont, dem Verbindungsglied der irdischen und himmlischen Sphäre, H. Brunner, Die Sonnenbahn in ägyptischen Tempeln, in: Archäologie und Altes Testament, Fs K. Galling, Tübingen 1970, $27-34$ und spez. $31 \mathrm{ff}$. sowie K. Jans en-Winkeln, Horizont und Verklärtheit: Zur Bedeutung der Wurzel 3h, in: SAK 23, 1996, $201 \mathrm{ff}$. 


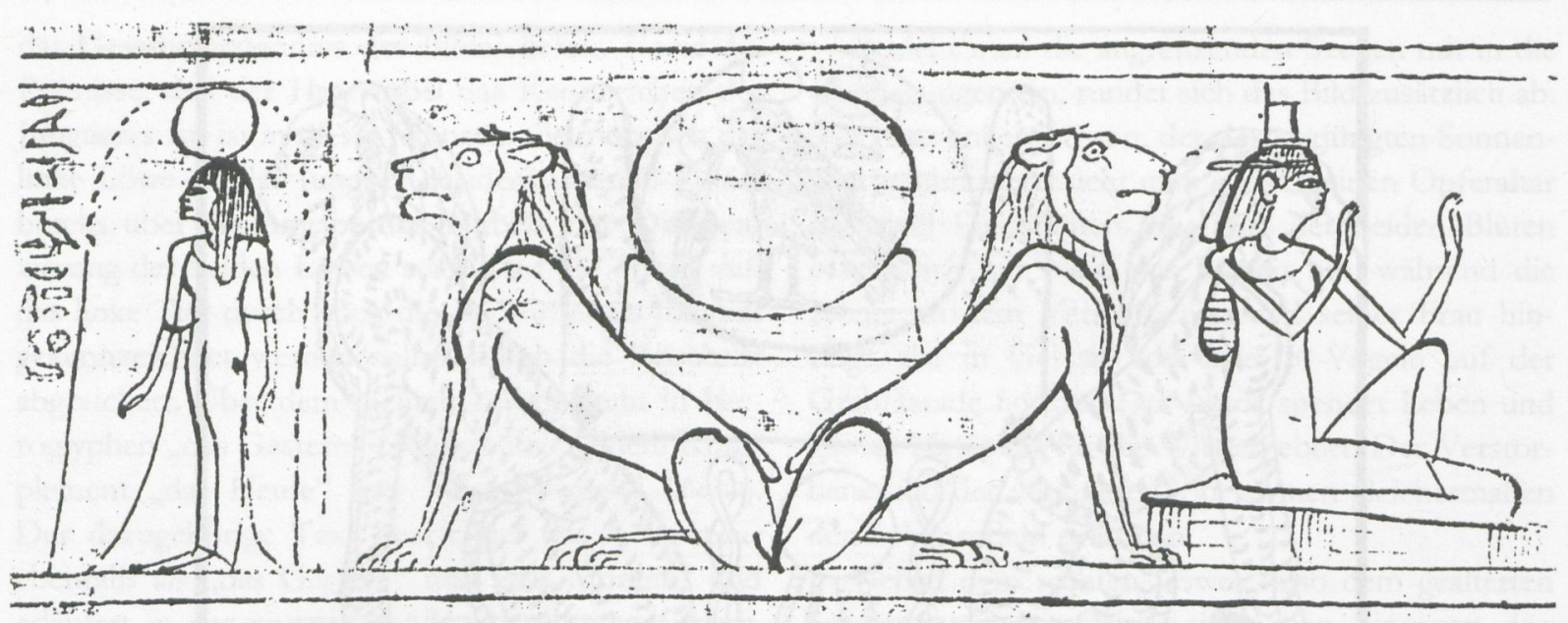

Abb. 2. Totenbuch der Ns-t3-nbt-jšrw (pGreenfield), Vignette zu Spruch 17

wieder unter ${ }^{33}$. Auch die Angabe der $n h h$-Ewigkeit des virtuellen Aspektes der Zeit ${ }^{34}$ - unter der Horizonthieroglyphe fügt sich harmonisch in dieses Bild ein, indem $n h h$ auf die zyklische Regeneration des Verstorbenen respektive des Sonnengottes und des Osiris Bezug nimmt. In diesem Zusammenhang ist die Überschrift des Totenbuchspruches $17 \mathrm{zu}$ zitieren, die resümiert, daß der Verstorbene in die Unterwelt hinabsteigt, mit Osiris verschmilzt und gleich dem Gott wiedergeboren wird ${ }^{35}$. Wie die Sonne, die am Abend in die Unterwelt hinabsinkt, um sich dort vorübergehend mit Osiris zu vereinen, möchte der Verstorbene mit Osiris eins sein. Sein Ziel ist es, zum

Siehe E. Hornung, Der Tempel als Kosmos, in: Geist der Pharaonenzeit, Zürich und München 1989, 115; J. Assmann, in: LÄ III, 5 mit Anm. 28, s. v. „Horizont“; cf. Westendorf, Darstellungen des Sonnenlaufes, 85.

${ }^{34}$ Zur Definition der beiden Zeitbegriffe $n h h$ (des virtuellen) und $\underline{d} t$ (des resultativen Aspektes der Zeit) sowie zu Re und Osiris und den beiden Zeitbegriffen $n h h$ und $\underline{d} t$ cf. J. Assmann, Stein und Zeit, München 1991, 52 (mit weiterer Literatur). Ferner A. Gutbub, Hathor hnt Twn.t, Rê Hor hnt Bhd.t, Amon hnt W3s.t, in: Mélanges Mariette, BdE 32, Le Caire 1961, 323f. (nhh als Himmel und Sonne und $\underline{d} t$ als Unterwelt und Mond). Zur Verbindung der $n h h$-Ewigkeit mit dem Morgen und der $\underline{d} t$ Ewigkeit mit dem Abend cf. De Morgan, Kom Ombos, 76: [Sobek] ist $n h h \underline{h}$ am Morgen und $\underline{d} t$ am Abend (lies: (8) $\Upsilon_{0}^{2} \approx$ ? Edfou I, 579, 5-6.

${ }^{35}$ Der Anfang des Spruches lautet „Anfang der Erhebungen und Verklärungen, des Herausgehens und (wieder) Hinabsteigens im Totenreich ... Herauszugehen am Tage, jegliche Gestalt anzunehmen ... als lebendiger Ba herauszugehen durch den NN, nachdem er bestattet wurde" (Hornung, Totenbuch, 59). vereinten $\mathrm{Ba}(b 3 d m \underline{d})$ zu werden und die Gegensätze aufzuheben $^{36}$.

In Totenbuchvignetten des pBM 10554 (pGreenfield), der vermutlich gegen Ende der 21. Dyn. niedergeschrieben wurde, haben sich zwei Darstellungen des Löwenpaares erhalten, von denen wieder jeweils das linke Tier den Haarwirbel an der Schulter aufweist und das rechte Tier nicht (Abb. 2) ( $^{37}$. In beiden Fällen tragen die Löwen die Horizonthieroglyphe auf dem Rücken. Im hier abgebildeten Beispiel ist neben dem linken Löwen die Besitzerin des Papyrus Ns-t3-nbt-jšrw zu sehen, die, ähnlich wie der Grabbesitzer in der soeben vorgestellten Szene im Grab des $N f r-3 b t$, rituell gereinigt wird. Rechts ist wie im Papyrus des $3 n j$ wieder die Totenbahre mit der Mumie der Verstorbenen dargestellt. An Kopf- und Fußende der Bahre hocken Nephthys und Isis mit erhobenen Händen. Auch in dieser Vignette blickt demnach der Löwe hinter Isis, der ohne den Haarwirbel dargestellt ist und in dem der gealterte Sonnengott zu erkennen ist, in Richtung der aufgebahrten Mumie des Verstorbenen.

${ }^{36}$ J. Assmann, Stein und Zeit, 52: „In Re und Osiris dagegen verbinden sich die Virtualität und die Resultativität nicht zu einer Weise des In-der-Zeit-Seins, sondern zur Zeit selbst. Re und Osiris sind nicht, in' Neheh und Djet, sondern sie sind Neheh und Djet." Die Zeit ist als duale Einheit aufzufassen. Der Sonnengott steigt als $\mathrm{Ba}$ in die Unterwelt hinab und vereint sich mit seinem Leichnam Osiris zu einem einzigen Gott ( $b 3 d m d$ ).

${ }^{37}$ Abb. nach Budge, op. cit., Taf. L und cf. Taf. IX (zu letzterer cf. das parallele Motiv auf Taf. XLI, das beide Löwen aber im Gegensatz hierzu ohne Wirbel zeigt). 
In einer zeitgleichen Vignette des mythologischen Papyrus der Hrjt-wbn sieht man das Sonnenkind im Uroboros. Es führt einen Finger der linken Hand an den Mund, ist an der Schläfe mit der Jugendlocke versehen und hält Krummstab und Geißel in der rechten Hand (Tafel XIX) ${ }^{38}$. Statt zwischen den Gipfeln des $\underline{d} w$-Gebirges hockt das Sonnenkind zwischen den Hörnern der Himmelskuh Methyer, von der nur der Kopf in Frontansicht zu sehen ist. Oben breitet sich ein Armpaar schützend um den Uroboros aus. Der rechte der beiden Löwen erhielt den Haarwirbel, der als dunkler Schatten auf dem Papyrus zu erkennen ist. Neben diesem rechten Löwen stehen ein Pavian ${ }^{39}$ und die Verstorbene im Adorationsgestus mit je einer Beischrift, die eine kurze Anrufung an den Sonnengott in seiner Barke am Neujahrstag enthält. In diesem Bild ist die Angabe des Haarwirbels ebenfalls sinnvoll und kennzeichnet den jungen Sonnengott. Darüber hinaus wird mit der Nennung des Neujahrstages nicht nur auf den täglichen, sondern auch auf den jährlichen Regenerationsprozeß Bezug genommen.

Im thebanischen Grab des $H^{\complement}-$ bhnt (TT 2) erscheint der Horizontberg in abweichender Bildkomposition an Stelle der Hathorkuh bzw. an Stelle der Sonnenscheibe, die aus dem Westgebirge aus eigener Kraft heraustritt oder von weiblichen Armen bewegt wird (Abb. 3) ${ }^{40}$. Das Horizontgebirge wird in diesem

${ }^{38}$ Tafel nach A. Piankoff, Myth. Pap., Taf. 1; cf. Hornung, Die Tragweite der Bilder, 233, Abb. 32 (der Wirbel wurde in der Zeichnung nicht berücksichtigt). Der Papyrus ist ebenfalls in die späte 21. Dynastie datiert. Zum Motiv des Sonnengottes, der im Gehörn der Himmelskuh sitzt, cf. zuletzt O. Keel und S. Schroer, in: ZÄS 125, 1998, 15 f. und weiterhin G. Englund, Propos sur l'iconographie d'un sarcophage de la 21e dynastie, in: Fs Säve Söderbergh, Boreas 6, Uppsala 1974, 54, Fig. 10 (= Darstellung der Horizontlöwen auf Kopfende der Sargwanne Uppsala 228); E. Hornung, Der ägyptische Mythos von der Himmelskuh, OBO 46, Freiburg und Göttingen 1982, 96-102. Ferner TT 336 (Nfr-rnpt): B. Bruyère, Fouilles de Deir el Médineh (1924-25), FIFAO 3, 3, Le Caire 1926, 101, Fig. 69.

S. Schott, Zum Weltbild der Jenseitsführer des neuen Reiches, in: NAWG Göttingen 1965, 86-87, identifiziert den Pavian mit dem Udjatauge als Thot.

${ }^{40} \mathrm{PM} \mathrm{I}^{2}, 1$, p. 9 (Decke). Siehe B. Bruyère, Tombes thébaines de Deir el Médineh à décoration monochrome, MIFAO 86, Le Caire 1952, Taf. 3. Bei der Darstellung der aus dem Gebirge heraustretenden Kuh oder Scheibe handelt es sich um die Vignette zu Tb 186. Cf. zu einem vergleichbaren Motiv H.Schäfer, Altägyptische Bilder der

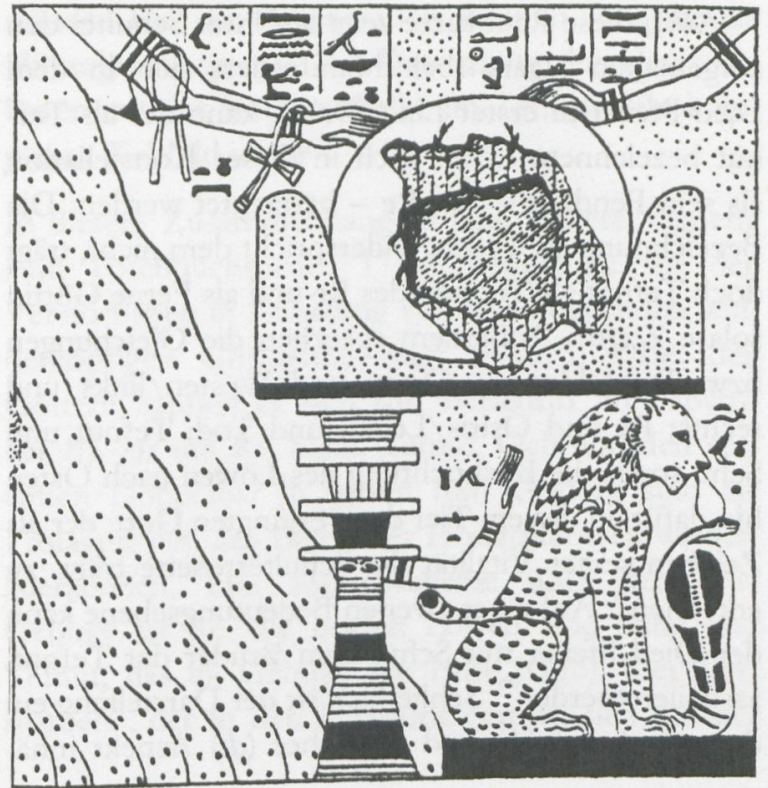

Abb. 3. Horizontgebirge im Grab des $H^{r}$-bhnt (TT 2)

Grab von einem Löwen und einem Djed-Pfeiler getragen. Der Löwe ist rechts und der Djed-Pfeiler links plaziert. In der Scheibe, die im Gebirge ruht, haben sich Reste von Umrissen eines Skarabäus, einer Erscheinungsform des Sonnengottes, erhalten. Darüber sieht man zwei menschliche Armpaare; das linke davon mit weiblicher Brust und Lebens- und Schutzzeichen an Handgelenk und Ellbogen sowie einer Beischrift, die wohl mit dem Namen der Himmelsgöttin Nut endet ${ }^{41}$. Vor dem hockenden Löwen bäumt sich eine Uräusschlange mit oberägyptischer Krone auf $^{42}$. Der Djedpfeiler besitzt aufgemalte Augen sowie einen menschlichen Arm, der im Schutzgestus im Rücken des Löwen erhoben ist. Vor dem Kopf des Löwen steht eine Beischrift, die „Tefnut“ lautet. Diesen Text möchte ich eher auf den Löwen als auf die Kobra beziehen ${ }^{43}$.

auf- und untergehenden Sonne, in: ZÄS 71, 1935, 34, Abb. 23.

${ }^{41}$ Alternativ wäre die Lesung $n t$ pt möglich. Die Ergänzung der Lücke ist unklar.

${ }^{42}$ Hornung, Die Tragweite der Bilder, 199-200, sprach der Uräusschlange und der Tefnut in der vorliegenden Bildkomposition eine apotropäische Funktion zu. Dazu wies er auf die den Sonnenlauf stets bedrohenden Gefahren hin, vor denen der Sonnengott geschützt werden mußte. Zugleich bemerkte Hornung aber den Widerspruch, daß diese Darstellungen in den Bildern der Horizontlöwen äußerst selten sind.

${ }^{43}$ Zur Löwengestalt der Tefnut cf. De Wit, Le rôle et le sens du lion, 324-332. Zur Sonnenscheibe, die bewegt wird, cf. die Darstellung in TT 336 (siehe Piankoff, 
Auch diese Raubkatze zeigt auf ihrer Schulter den aufgemalten Haarwirbel. Manifestiert sich in dem Djed-Pfeiler in erster Linie Osiris, kann der als Tefnut bezeichnete Löwe auch in dieser Konstellation als sein Pendant - also Re - betrachtet werden. Die Bezeichnung als Tefnut widerspricht dem nicht, trägt doch Tefnut als Tochter des Re und als Ferne Göttin solare Züge $e^{44}$. Außerdem sprechen die Gleichungen bzw. Paarbildungen Osten und Westen/links und rechts; Re und Osiris/Leben und Tod; Tefnut und Schu sowie die Blickrichtung des Löwen nach Osten hin dafür, in diesem Tier den verjüngten Gott, der als Zeichen seiner Vitalität die Schulterrosette trägt, zu erkennen ${ }^{45}$. Auf einer zweiten Bedeutungsebene kann der Djed-Pfeiler mit Schu, dem Bruder der Tefnut, assoziiert werden ${ }^{46}$. Schließlich ist der Darstellung ein dynamischer $(n h h)$ und statischer $(\underline{d} t)$ Aspekt inhä-

Myth. Pap., 41, Taf. 25). M. Herb und U. Verhoevenvan Elsbergen wiesen mich darauf hin, daß sich der Text auch auf die Kobra beziehen könnte. Vielleicht gehört die Uräusschlange aber auch zur Ikonographie der löwengestaltigen Tefnut; dann bezöge sich die Beischrift auf beide (Hinweis $\mathrm{Ph}$. Derchain). Für diese Überlegung mag sprechen, daß Löwe und Schlange auf einer gemeinsamen Standlinie (Podest) abgebildet sind, während der Djedpfeiler ohne Sockel direkt im Westgebirge (dem unterweltlichen Bereich) steht.

${ }^{44} \mathrm{Zu}$ Tefnut als Löwin und dem Mythos vom fernen Sonnenauge s. U. Verhoeven, in: LÄ VI, $297 \mathrm{ff}$., s. v. „Tefnut" und cf. stellvertretend eine Darstellung im Tempel von Dakke (Roeder, Dakke II, Taf. 115), die eine schreitende Löwin mit Sonnenscheibe und Uräus auf dem Kopf zeigt. Vor ihr steht ein Pavian im Anbetungsgestus.

${ }^{45}$ Anders W. Westendorf, Die geteilte Himmelsgöttin, in: I. Gamer-Wallert und W. Helck (Hrsgg.), Festschrift für E. Brunner-Traut, Tübingen 1992, 341 ff., der Tefnut zwar mit dem Löwenvorderteil $(h 3 t)$ in Beziehung setzt, aber in ihr die Raubkatze erkennt, die am Abend die Sonne verschlingt, während er Schu als Himmelserheber mit dem Löwenhinterteil $(p h)$ und dem Osthorizont gleichsetzt.

${ }^{46} \mathrm{Zu} d d$ und $w 3 s$ als Bezeichnungen von Schu und Tefnut in ptolemäischer Zeit siehe E. Winter, Untersuchungen zu den ägyptischen Tempelreliefs der griechischrömischen Zeit, DÖAW 98, Wien 1968, 85-88. - Cf. in diesem Zusammenhang vielleicht auch eine Ritualszene der Übergabe von ' $n h$ und $w 3 s$ in der Wabet des Tempels von Edfu (Edfou I, 425, 2-9). In der königlichen Randzeile dieser Szene ist der König als Erbe des Schu, geboren von Tefnut ausgewiesen. Der Text zu Horus Behedeti, dem Empfänger des Rituals, beginnt interessanterweise

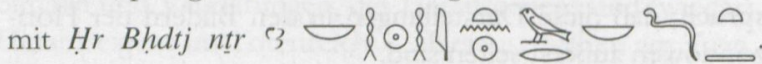
Bei dieser Abfolge der Epitheta hat man wieder das Bild der Horizontlöwen mit der Sonnenscheibe in ihrer Mitte vor Augen. rent. Osiris oder Schu als Djed-Pfeiler im Gebirge steht im Kontrast zur aus dem Berg bereits herausgetretenen, in Bewegung befindlichen Tefnut als Sonne. Vor diesem Hintergrund symbolisieren die Horizontlöwen nicht nur die tägliche, sondern auch die jährliche Regeneration. Das Bild bietet dem Betrachter mehrere Möglichkeiten der „Lesung“ und führt ihn ,von Assoziation zu Assoziation weit über die engeren Grenzen des Motivs hinaus ${ }^{647}$.

Als letztes Beispiel für die Horizontlöwen sei auf eine Adaption dieses Motivs auf einem Sarg aus der 21. Dynastie verwiesen, der sich heute im Museum in Kairo befindet. Dort flankieren die Löwen, die diesmal Maat-Federn in den Vorderpranken halten, einen Djed-Pfeiler (Abb. 4) ${ }^{48}$. Dieser besitzt menschliche Arme und hebt mit ihnen eine Barke empor. In der Barke ist eine von zwei Udjataugen umschirmte Scheibe zu sehen. Auf der Schulterpartie des rechten Löwen ist in der Publikationszeichnung ein runder Fleck angegeben, der wohl den Wirbel darstellen soll.

Das Bild der Rücken an Rücken hockenden Horizontlöwen symbolisiert den immerwährenden Kreislauf der auf- und untergehenden Sonne und zugleich den zyklischen Tod und die Regeneration des Sonnengottes und des Osiris. Die über dem Rücken der Tiere, dem Verbindungsglied zwischen dem Löwenvorderteil (Anfang: hit कु ) und dem Hinterteil (Ende: $p h$ SU), schwebende Horizonthieroglyphe konnotiert die $n h h$-Zeit ${ }^{49}$. Vereint sich der Verstorbene, wenn er am Tage aufgeht, mit $n h h(\mathrm{Re})$ und bei

Siehe Hornung, Die Tragweite der Bilder, 197 Hornung bemerkt dies bezüglich der „Beiwerke“ in den Bildern. Zu diesen „Beiwerken“ zähle ich auch die Haarwirbel.

${ }^{48}$ CG $6008=$ E. Chassinat, La seconde trouvaille de Deir el-Bahari (sarcophages), Leipzig 1909, 26, Fig. 24. $\mathrm{Zu}$ den Löwen mit Maat-Federn cf. die Darstellung der Horizontlöwen mit Federn auf dem Kopf im pBM 10018 (s. die Abb. bei S. Schott, Zum Weltbild der Jenseitsführer des neuen Reiches, in: NAWG Göttingen 1965, 192 , Taf. 4) und bei A. Niwiński, Studies on the Illustrated Theban Funerary Papyri of the 11th and 10th Centuries B.C., OBO 86, Freiburg/Göttingen 1989, Taf. 48 b. Cf. auch Hornung, Die Tragweite der Bilder, 197 und 226, Abb. 17 (= Sarg Kairo 1238).

49 Zur Lesung der Horizonthieroglyphe (mit oder ohne Sonnengott in der Scheibe) in der griech.-röm. Zeit als nhh-Ewigkeit cf. z. B. Edfou III, 105, 11; 106, 6; IV, 352, 8 oder Dendara II, 152,7 und IV, 104,13. - An das Bild der Horizontlöwen erinnern die Hieroglyphen 2 ? und 3. 3 . Das Zeichen ohne die Sonnenscheibe kann als Gott 


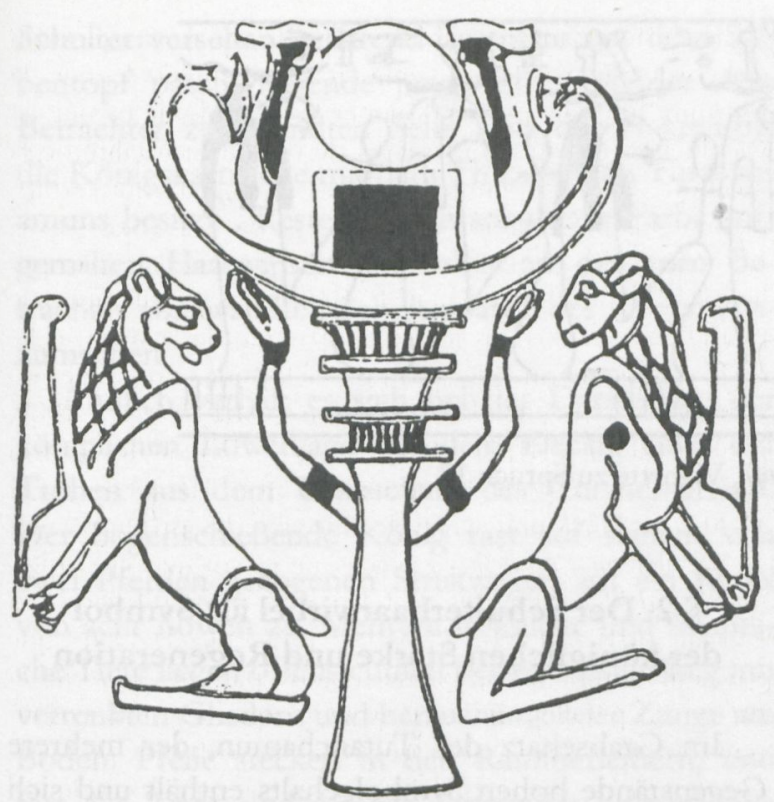

Abb. 4. Löwendarstellung auf dem Sarg CG 6008

seinem Eintritt in die Nacht mit $\underline{d} t$ (Osiris) $^{50}$, so wird er in den Totenbuchvignetten in den kosmischen Kreislauf auf das kunstvollste eingebunden. Er kann vor dem linken Löwen, der mittels des Schulterhaarwirbels als der kräftige, verjüngte Gott gekennzeichnet ist, im Anbetungsgestus stehen und vergleichbar dem gestärkten Sonnengott, der die Gefahren der nächtlichen Reise durch die Finsternis siegreich über-

Aker gelesen werden (zu Schreibungen des Aker mit dem Löwenpaar siehe S. Hassan, Gîza VI, I, 257-276 sowie 'De Wit, Le rôle et le sens du lion, 91-106) und daneben, sowohl mit als auch ohne Sonnenscheibe, den Lautwert hns besitzen (zur Lesung hns siehe Wb III, 299, 5-19 („durchziehen“) und cf. Wb III, 300, 5 (ein Amulett; cf. dazu Petrie, Amulets, 45 und Taf. 39, Nr. 220 c) und Wb III, 300, 6 (eine Tür). Es erscheint ferner als Schreibung des Namens des Chons und steht somit nicht nur mit dem Sonnenlauf, sondern auch mit dem Mondzyklus in Beziehung (siehe z. B. Edfou I, 56,5; Edfou I, 378, 10; De Wit, Opet I, 15). Ähnlich wird der Doppelstier "ȳk hns (,durchziehen “) gelesen (cf. Petrie, Amulets, p. 45 und Taf. 39, Nr. 223 a zu einem Doppelstieramulett). Wie die Löwen steht er als Schriftzeichen mit dem Sonnenlauf in Zusammenhang. Aus einer Stelle in den Sargtexten geht hervor, daß der große Doppelstier am Scheitel des Re ist und das Leuchten im Osten bewirkt (CT III, $381 \mathrm{a}$; cf. $400 \mathrm{a}$ und $\mathrm{cf}$. ferner CT III, 3a). Bei den Widdervorderteilen ist gelegentlich eine antithetische Anordnung belegt. Siehe dazu Edfou I, 343, 5: B $3 t j$ befindet sich am 20. o.äg. ist Gau als I, 25,18 .

${ }^{50}$ Cf. F. R. Herbin, Le livre de parcourir l'éternité, OLA 58, Leuven 1994, I, 14. standen hat, die Regeneration erbitten. Vor diesem Hintergrund spiegelt die Darstellung der Horizontlöwen mit der Sonnenscheibe im Zentrum ein komplexes Weltbild wider ${ }^{51}$.

In diesem Zusammenhang ist eine weitere Vignette zum Totenbuchspruch $17 \mathrm{zu}$ betrachten, die den Verstorbenen in Anbetung vor fünf hockenden widderköpfigen Gottheiten zeigt (Abb. 5) ${ }^{52}$. Bei den Göttern handelt es sich laut Beischrift im Papyrus des Hw-nfr um Re, Schu, Tefnut, Geb und den vereinten Doppelba. In diesen fünf Göttern sind die Protagonisten des Bildes der Horizontlöwen wiederzuerkennen. Die Sonnenscheibe über dem $\underline{d} w$ Gebirge ist Re, die beiden Löwen sind Schu und Tefnut, das $\underline{d} w$-Gebirge die Erde (Geb). Der Verstorbene, der in den Horizont eintritt, sich mit der

${ }^{51}$ Cf. J. Assmann, in: LÄ III, 5, s. v. „Horizont“. Cf. vielleicht ferner zu Schu und Tefnut mit dem Sonnengott in ihrer Mitte einen Block aus Musawwarat es Sufra, der sich heute im Berliner Ägyptischen Museum befindet (Inv. Nr. 24300). Es handelt sich um eine Dreiergruppe, die in der Mitte den widderköpfigen Amun mit Federkrone und Doppeluräus über einer Lotosblume zeigt. Flankiert wird er von zwei Löwen, die jeweils die $\mathrm{hmhm}$ Krone auf dem Kopf tragen, s. die Abb. bei K.-H. Priese (Hrsg.), Ägyptisches Museum Berlin, Mainz 1991, 261 (Nr. 159). I. Hofmann äußerte die Vermutung, daß die beiden Löwen Schu und Tefnut darstellen (siehe I. Hofmann, Zur Darstellung von Schu und Tefnut im meroitischen Reich, in: GM 23, 1977, 45-47), während sich St. Wenig, in: ZÄS 101, 1974, 130 ff. mit Abb. auf Taf. VII a, für Darstellungen der nubischen Götter Arensnuphis und des Sebiumeker aussprach. Im Hinblick auf die oben gemachten Äußerungen zu den Horizontlöwen ist die Theorie, in den beiden Tieren Schu und Tefnut wiederzuerkennen, verlockend. Dies wird jetzt auch im von D. Wildung herausgegebenen Katalog, Die Pharaonen des Goldlandes. Antike Königreiche im Sudan. Ausstellungskatalog Mannheim 1996, 279, angenommen, wobei die Mondsichel des rechten Löwen noch einer Erklärung bedürfte. Auch in diesem Fall würden die Götter den aufsteigenden Sonnengott in ihrer Mitte umschirmen.

52 Abb. nach Tb (Naville), Taf. 30, A. g. Cf. E. Hornung, Totenbuch, 68-69. Im Text heißt es dazu (\$ 21) „Ich bin sein Doppel-Ba, der in seinem Doppelträger ist. Was bedeutet das? Osiris ist das, als er in Mendes eintrat, dort fand er den Ba des Re. Da umarmte einer den anderen, da wurden (sie) zu seinem Doppel-Ba“. H. Milde, The Vignettes of the Book of the Dead of Neferrenpet, Egyptologische Uitgaven VII, Leiden 1991, 38, weist darauf hin, daß Geb im Text des Spruches nicht erwähnt wird. Das „sic“ von Milde wird relativiert und vielleicht hinfällig, wenn man in dem $\underline{d} w$-Gebirge bzw. dem im Text genannten Löwenpaar (Ruti oder Aker) den Erdgott Geb erkennt. 


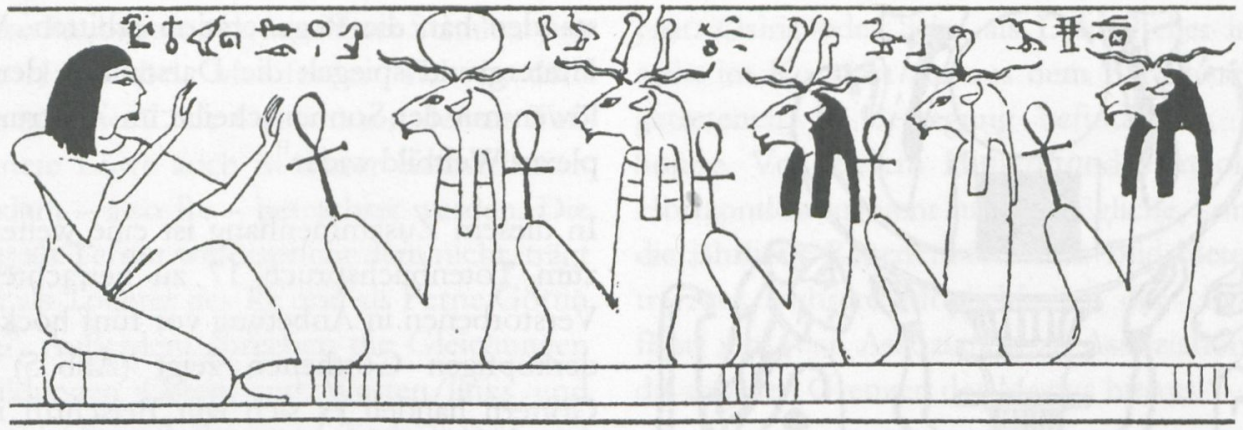

Abb. 5. Totenbuch des Hw-nfr, Vignette zu Spruch 17

Nachtsonne vereint und am Morgen wiedergeboren wird, möchte zu Osiris werden, dem vereinten $\mathrm{Ba}$. Gleichzeitig ist dem Bild der Horizontlöwen die Vorstellung von den vier Elementen immanent. Geb steht für das Land, Re für das Licht, Schu für die Luft und Tefnut für das Wasser ${ }^{53}$.

In späten Tempeltexten könnte das hier gezeichnete Bild fortleben. In einer der beiden ChonsKapellen im Tempel von Edfu ist beispielsweise zu lesen, daß Chons-Schu den Himmel erhebt und die 3ht des Himmels geheim macht (sšt 3$)$. Er gibt außerdem die Bas der Götter in sein (scil. des Horizonts) Inneres ${ }^{54}$. Hathor wird in dieser Szene als Tefnut bezeichnet, die sich mit ihrem Bruder vereint, um den Leib des Erzeugers/Sohnes wohlbehalten sein zu lassen. Hatte hier der antike Textverfasser bei der Erwähnung der Bas, die sich im Inneren des Horizontes befinden, die widderköpfigen Götter der Totenbuchvignette im Gedächtnis, vor denen der Verstorbene im Anbetungsgestus hockt und in denen sich Re, Schu, Tefnut, Geb und der vereinte Ba (Osiris) manifestieren? Und verbirgt sich weiter hinter der Aussage, daß Hathor-Tefnut sich mit ihrem Bruder vereint, das Bild der beiden Horizontlöwen Schu und Tefnut, die Rücken an Rücken den Sonnengott tragen und ihn wohlbehalten sein lassen?

Cf. dazu die Erwähnung des Ba mit den vier Köpfen auf einem Hals, den Assmann mit den vier Elementen in Beziehung setzt (J. Assmann, Re und Amun, OBO 51, Freiburg 1983, 262f. und ders., Sonnenhymnen in thebanischen Gräbern, Mainz 1983, 207, Anm. t). Cf. ferner Chr. Leitz, Tagewählerei, $426 \mathrm{f}$.

${ }^{54}$ Edfou I, 279,3-6 (cf. die Parallele in Edfou II, 57,2 und vergleichbare Textstellen bei A. Gutbub, Textes fondamentaux de la théologie de Kom Ombo, BdE 48/1, Le Caire 1973, 87-88).

\section{$\$ 2$ Der Schulterhaarwirbel als Symbol der königlichen Stärke und Regeneration}

Im Grabschatz des Tutanchamun, der mehrere Gegenstände hohen Symbolgehalts enthält und sich besonders für Studien zur Ikonographie eignet ${ }^{55}$, finden sich Darstellungen von Löwen mit der Angabe des Haarwirbels an der Schulter auf unterschiedlichen Objekten.

Auf dem Salbgefäß aus Alabaster, auf dessen Dekkel die rundplastische Figur eines Löwen mit übereinandergeschlagenen Vorderpranken ruht, sind als Kontrapost an den Außenwänden Szenen abgebildet, in denen Wildstiere von Löwen angegriffen werden ${ }^{56}$. Man sieht an der Vorderseite einen Löwen, der auf den Rücken eines Stieres gesprungen ist, diesen mit seinen Pranken fest umklammert und zum tödlichen Biß in den Nacken des Stieres ansetzt. Dieses angreifende, aggressive und kraftvolle Raubtier hat der altägyptische Künstler mit einem Haarwirbel an der

Zum Symbolgehalt einiger Objekte des Grabschatzes des Tutanchamun cf. F. Abitz, Statuetten in Schreinen, AA 35, Wiesbaden 1979, 89 (Statuetten des Königs auf Panther); D. Kurth, Zur Erklärung eines Bildmotivs auf einem der Prunkohrringe Tutanchamuns, in: Z ̈̈S 109, 1982, 62-65; E. Graefe, Der Sonnenaufgang zwischen den Pylontürmen, in: OLP 14, 1983, 55-79 (zur $h s$-Vase zwischen den Pylontürmen); E. Feucht, Verjüngung und Wiedergeburt, in: SAK 11, 1984, 408-410 (zum kartuschenförmigen Salbgefäß JE 61496, das den König in drei verschiedenen Lebensphasen zeigt und auf die zyklisch wiederkehrende Geburt aus dem Urgewässer anspielt); J. Qua egebeur, La naine et le bouquetin ou l'énigme de la barque en albâtre de Toutankhamon, Leuven 1999 (zur Symbolik der Alabasterbarke JE 62120).

${ }^{56}$ Kairo JE 62119. Siehe die Abb. im Ausstellungskatalog „Tutanchamun“, Hannover 1981, Nr. 49 und zu beiden Seiten des Gefäßes siehe I. E. S. Edwards, Treasures of Tutankhamun, New York 1976, Taf. 19 (Schauseite) und 124 (Rückseite). 
Schulter versehen, während der oben auf dem Salbentopf passiv liegende junge Löwe an der dem Betrachter zugewandten Seite statt der Haarwirbel die Königskartusche mit dem Thronnamen Tutanchamuns besitzt ${ }^{57}$. Reste eines in schwarzer Farbe aufgemalten Haarwirbels sind aber an der vom Betrachter abgewandten Schulterpartie des Löwen auszumachen.

Ähnlich verhält es sich bei der Darstellung der königlichen Löwenjagd auf dem Deckel einer der Truhen aus dem Grabschatz des Tutanchamun ${ }^{58}$. Der bogenschießende König rast auf seinem von zwei Pferden gezogenen Streitwagen auf ein Rudel von acht Löwen zu. Mehrere weibliche und männliche Tiere liegen offensichtlich bewegungsunfähig mit verrenkten Gliedern und heraushängender Zunge am Boden. Pfeile stecken in den Raubtierleibern, und fast alle Tiere sind schwer verwundet oder tödlich getroffen. Nur ein Löwe stürmt aus der hinteren Reihe unerschrocken und angriffslustig mit weit aufgerissenem Maul, drohend herausgestreckten Krallen und erhobenem Schwanz auf den Streitwagen des Königs los. Als einzigem des Löwenrudels hat ihm der altägyptische Künstler deutlich den Haarwirbel auf die Schulter gemalt und ihn dadurch als das Tier gekennzeichnet, das furchtlos und noch im vollen Besitz seiner Kräfte ist, während seine

${ }^{57}$ Es wurde vermutet, daß der Löwe auf dem Deckel den König verkörpert (Chr. Desroches-Noblecourt, Tut-ench-Amun, Berlin, Frankfurt und Wien 1963, 210). Trifft diese Vermutung zu, wären auf diesem Gefäß zwei Aspekte des Herrschers dargestellt, nämlich der des jungen friedlichen und der des jungen kämpferischen. Ferner mag die Tatsache, daß die Königskartusche an die Stelle des Haarwirbels getreten ist, eine weitere assoziativ zu lesende Information beinhalten. Denn durch die Positionierung der Kartusche an ebender Stelle, an der die Vitalität des Tieres markant zum Ausdruck kommt, könnte die Macht und Stärke, die dem königlichen Namen und seinem Träger anhaftet, zusätzlich betont worden sein.

${ }^{58}$ Kairo JE 61467. Siehe die Abb. bei N. M. Davies und A. Gardiner, Tut-ankh-amun's Painted Box, Oxford 1962, Taf. IV; ferner W. Decker und M. Herb, Bildatlas zum Sport im Alten Ägypten, HdO 14, 1, Leiden, New York, Köln 1994, 345-346, Nr. J121 b. Zentrales Thema auf der Truhe ist der Triumph des Königs über die wilde Natur und damit die Bezwingung des Chaos. U. Schweitzer, Löwe und Sphinx im Alten Ägypten, ÄgFo 15, 1948, 55-56, weist auf die auffallend naturalistische Wiedergabe des Jagdgetümmels hin und bemerkt zum Kampf zwischen dem König und dem Löwen: „Die Größe eines Herrschers wächst mit der Größe seines Widersachers“. - Zur Löwenjagd cf. W. Decker, Die physische Leistung Pharaos, Köln 1971, 44-52.
Artgenossen schwer verletzt oder bereits verendet $\sin ^{59}$.

Obwohl die Darstellung der Löwenjagd natürlich ein idealisiertes Bild der Wirklichkeit wiedergibt, zeigt doch vor allem das Beispiel der Löwenjagd des Tutanchamun, daß das Motiv des Schulterhaarwirbels bewußt angebracht ist, um eine Aussage zu intendieren, die dahin zielt, die Stärke des Tieres, mit der sich der König markanterweise auf gleicher Höhe, d. h. Auge in Auge, mißt und die deshalb seine eigene Kraft unterstreicht, nicht nur vordergründig bildlich auszudrücken, sondern auch im kleinen Detail wirksam werden zu lassen ${ }^{60}$.

Die Elfenbeinkopfstütze des jungen Tutanchamun, auf welcher dieser ruhen sollte, um am nächsten Morgen regeneriert und in seiner königlichen Macht erneut gestärkt zu erwachen, zeigt zwei liegende Löwen, welche jeweils auf beiden Schultern den Haarwirbel besitzen ${ }^{61}$. Beide Löwen flankieren den menschengestaltigen Schu, der die halbmondförmige Stütze trägt und dessen Arme dabei von

59 Möglicherweise trägt noch ein weiterer Löwe des Rudels den Haarwirbel. Es handelt sich bei dem in Frage kommenden Tier um den jungen Löwen, der in der linken unteren Ecke des Bildfeldes flüchtet. Er ist wohl auf seiner linken (für Betrachter nicht sichtbaren) Flanke von einem Pfeil getroffen. Anhand der Publikationsphotos ist eine sichere Entscheidung nicht möglich. - Zu weiteren Darstellungen von Löwen mit dem Schulterhaarwirbel bei der Jagd in der freien Wildnis cf. Ostrakon Kairo IAO 3005 (s. die Abb. bei W. Forman und H. Kischkewitz, Die Altägyptische Zeichnung, Hanau 1971, Taf. 6), eine Elektron-Plakette aus Ras Schamra aus der Zeit des NR, die sich heute im Louvre befindet (s. die Abb. bei J. Leclant (Hrsg.), Universum der Kunst, Ägypten, Bd. II, Das Großreich, München 1980, 293, Nr. 332) oder die Darstellung eines Löwen mit Schulterhaarwirbel auf einem in Semna gefundenen Dolch aus der Zeit des Neuen Reiches (s. die Abb. bei D. Wildung, Die Pharaonen des Goldlandes. Antike Königreiche im Sudan. Ausstellungskatalog Mannheim 1996, 134-136, Nr. 138).

${ }^{60}$ Die Beischrift des Königs (siehe H. Beinlich und M. Saleh, Corpus der hieroglyphischen Inschriften aus dem Grab des Tutanchamun, Oxford 1989, 7 = Carter, Nr. 21) erläutert die Darstellung. Der König trägt das Epitheton „der mit starker Kraft“ (shm phtj) und ist der, „der die Löwen bekämpft“" ( $h 3$ m $m$ 3jw).

${ }^{61}$ Kairo JE 62020. Siehe die Abb. im Katalog „Tutanchamun", Hannover 1981, Nr. 26. Die Kopfstütze symbolisiert daneben das Horizontgebirge, aus dem der junge Sonnengott am Morgen gestärkt hervorkommt. Die Löwen können auch das „Gestern" und das „Morgen“, das West- und das Ostgebirge oder Schu und Tefnut verkörpern. Cf. ferner den Totenbuchspruch für die Kopfstütze (Tb 166). 


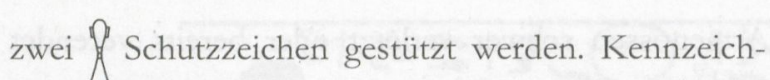
net der Haarwirbel das junge, kraftstrotzende Tier, so ist die Angabe des Wirbels auch auf diesem Bildträger sinnvoll, setzt man zum einen die Funktion des Löwen als Beschützer des Schlafenden an und geht man zum anderen von der Prämisse aus, daß die Angabe der Haarwirbel die Jugend und Stärke der Raubtiere kenntlich machen und bewirken soll, daß diese Eigenschaften der Tiere während seines Schlafes auf den König übergehen ${ }^{62}$.

Bei der bekannten Darstellung des jugendlichen Königspaares während der Vogeljagd auf dem goldenen Statuenschrein des Tutanchamun ist neben dem bogenschießenden, auf einem Hocker sitzenden König ein schreitender junger Löwe mit Halsband und demnach wahrscheinlich ein gezähmtes Tier abgebildet ${ }^{63}$. Auf der Schulter des Löwen ist der Haarwirbel angegeben. Auch dieses Beispiel reflektiert das Bestreben des Künstlers, die königliche Vitalität selbst im winzigen Detail transparent zu machen. In diesem Zusammenhang ist ferner von Bedeutung, daß für die Darstellung des Königspaares

${ }^{62}$ Cf. zu dieser Doppel-Funktion (Schutz und Regeneration) der Kopfstützen zum einen die AR-Kopfstütze Berlin 20462 (s. die Abb. im Katalog, Anch', Blumen für das Leben, Pflanzen im Alten Ägypten, S. Schoske, B. Kreiß1 und R. Germer (Hrsgg.), München 1992, Kat. Nr. 123). Bei dieser Kopfstütze wird die halbrunde Liegefläche von einer kannnelierten Säule in der Mitte sowie zwei Lotos-Pflanzensäulen an den Außenseiten getragen. Der Lotos gilt gleichfalls als Symbol der Auferstehung und Verjüngung. Eine Schutzfunktion der beiden Träger der Stütze wird dagegen an der Kopfstütze Kairo CG 65832 aus Deir el Medineh erkennbar (s. $\mathrm{B}$. Bruyère, Rapports sur les fouilles de Deir el-Médineh (1934-35) FIFAO 16, Le Caire 1939, 229, Fig. 118. Dort wird die Liegefläche von zwei Dämonen flankiert, die böse Geister vom Ruhenden fernhalten sollen. Der eine Wächter ist löwengestaltig, steht mit den Vorderläufen auf zwei Schutzzeichen, ist mit Messern bewaffnet, und aus seinem Maul treten Schlangen hervor. Der zweite ist canidenköpfig, im Laufschritt mit einer Schlange (?) in der Hand dargestellt, und aus seinem Kopf winden sich ebenfalls Schlangen. Vor dem Hintergrund dieser regenerativen Kräfte sind außerhalb des Grabschatzes des Tutanchamun auch die Löwenbahren, auf denen die Mumie des Verstorbenen ruht, zu betrachten.

${ }^{63}$ Kairo JE 61481. Siehe die Abb. bei M. EatonKrauss und E. Graefe, The Small Golden Shrine from the Tomb of Tutankhamun, Oxford 1985, Taf. 15 und 36-37. bei der Vogeljagd eine Regenerationssymbolik postuliert wurde ${ }^{64}$.

Das Motiv des Löwen mit dem Haarwirbel auf der Schulter neben dem Königsthron läßt sich auch außerhalb des Grabschatzes des Tutanchamun belegen $^{65}$. Ein in ramessidische Zeit datiertes Ostrakon zeigt ebenfalls einen Löwen mit Schulterhaarwirbel neben dem Königsthron (Abb. 6) ${ }^{66}$. Auf diesem Ostrakon hat der Zeichner den Entwurf eines Königs angefertigt, der die Blaue Krone mit dem Uräus auf dem Kopf trägt und mit Flagellum und Krummstab in den Händen auf einem Tragsessel thront. Der Tragsessel ist an den Außenseiten der Armlehne mit der Darstellung eines Sphinx verziert, dessen Kopf mit der Doppelkrone geschmückt ist. Neben dem Thron schreitet majestätisch ein lebendiges Tier, das

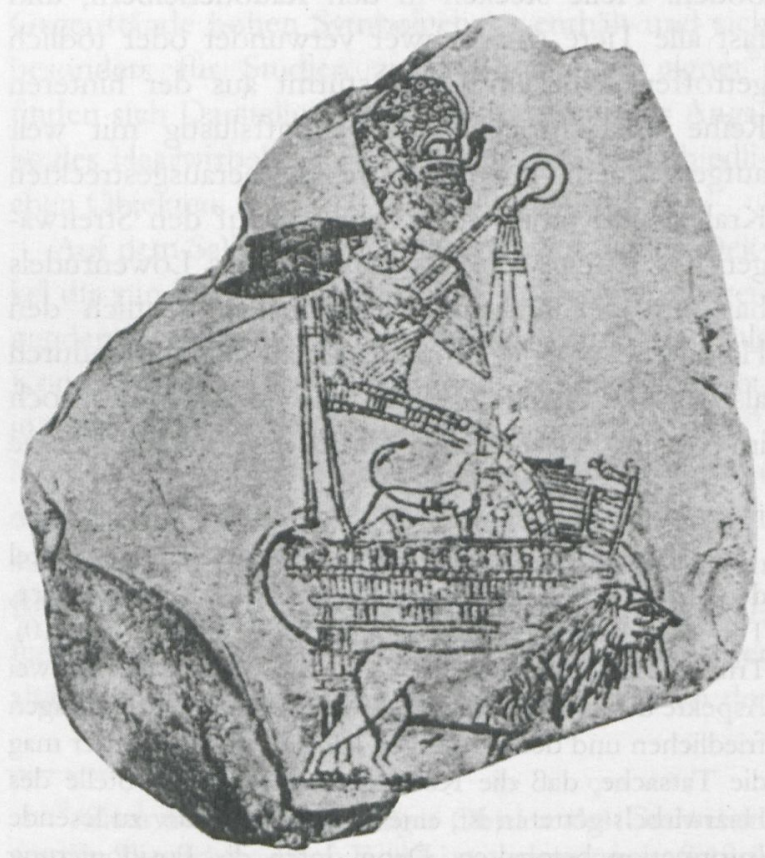

Abb. 6. Darstellung eines Löwen auf einem Ostrakon

${ }^{64}$ Zur darüber hinaus erotischen Symbolik der Szene cf. W. Westendorf, Bemerkungen zur ,Kammer der Wiedergeburt ${ }^{\star}$ im Tutanchamungrab, in: ZÄS 94, 1967, $142-144$

${ }^{65}$ Zum Motiv des Löwen, der neben dem Thron des Königs schreitet, siehe auch M. Metzler, Königsthron und Gottesthron, AOAT 15, 2 Bde., Neukirchen - Vluyn 1985, Bd. I, $10 \mathrm{ff}$, und speziell Nr. 271 (Ramses III; die Schulterpartie des Löwen ist leider zerstört) und die Stele MFA Boston CAA 25.632 (siehe Helck, in: JNES 14, 1955, $22 \mathrm{ff}$;; die Schulterpartie des Löwen ist ebenfalls zerstört).

${ }^{66}$ Das Stück befindet sich heute im Ägyptischen Museum in Berlin. Siehe die Abb. bei W. Kaiser (Hrsg.), Ägyptisches Museum Berlin, Berlin 1967, Nr. 720. 


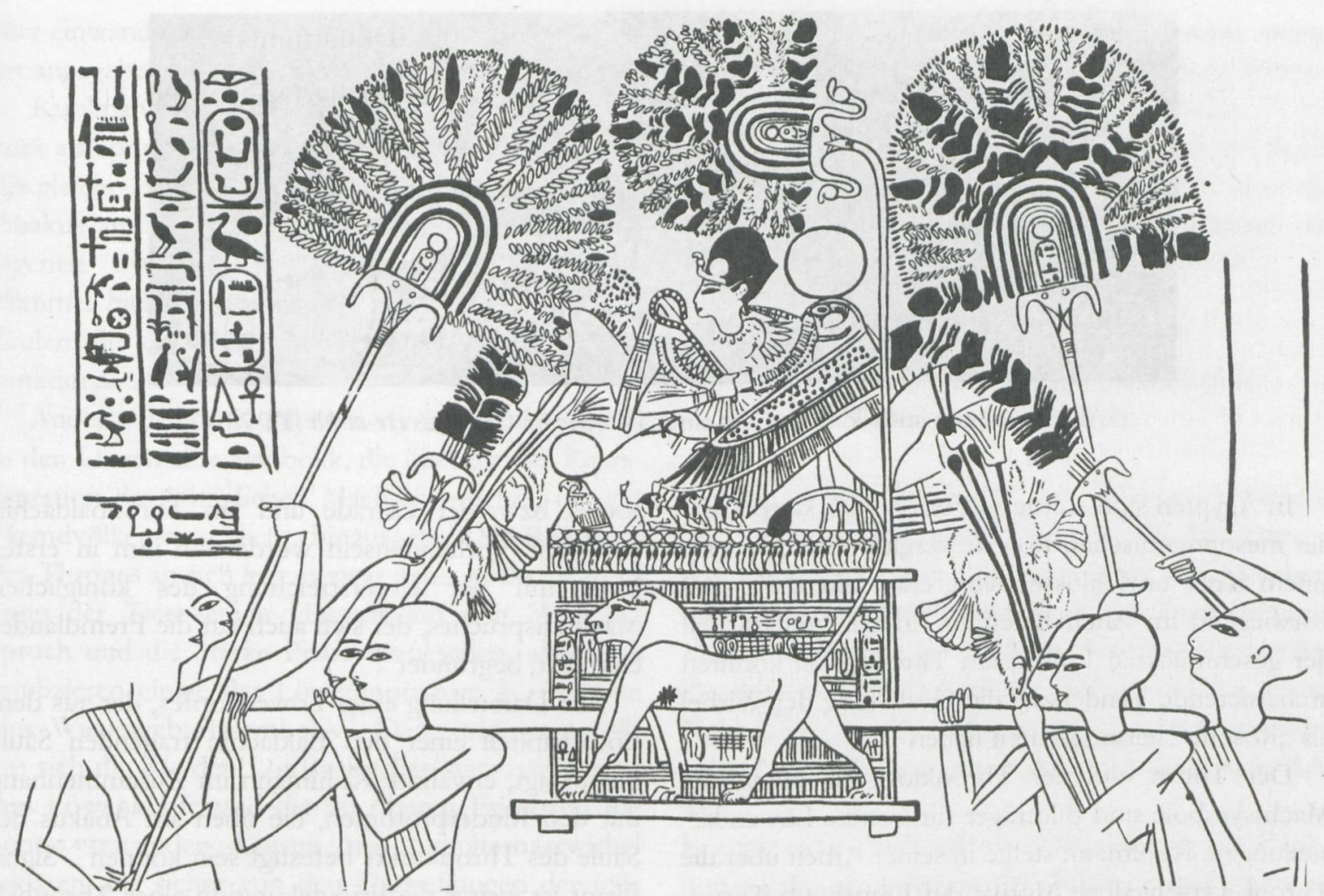

Abb. 7. Darstellung eines Löwen im Grab des Jmn-ms (TT 19)

einen überdimensional proportionierten Haarwirbel an der Schulter besitzt.

Eine vergleichbare Darstellung aus früherer Zeit hat sich fragmentarisch im thebanischen Grab des Jmn-ms (TT 19) erhalten (Abb. 7) ${ }^{67}$. Amenophis I. wird dort, mit seinen Herrschaftsinsignien ausgerüstet, in einer Prozession von mehreren Priestern im Tragsessel getragen. Neben dem Königsthron schreitet auch hier ein Löwe mit erhobenem Schwanz, und seine Schulter ist ebenfalls mit dem Haarwirbel verziert.

Bei den soeben angeführten Beispielen handelt es sich wahrscheinlich um die Darstellung domestizierter junger Raubtiere. In diesem Zusammenhang ist Jequier $^{68}$ zu zitieren, der als erster davon ausging, daß

Siehe G. Foucart, Tombes Thébaines, Le Tombeau d'Amonmos, MIFAO 57, Le Caire 1932, Taf. 28 (= Wandmalerei im Grab TT 19; heute rekonstruiert; Zeichnung nach Hay).

${ }^{68}$ G. Jequier, Les pyramides des reines Neit et Apouit, in: Fouilles à Saqqarah, Le Caire 1933, 7. Diese Theorie wurde später aufgegriffen von Kantor, op. cit., 253, n. 18. - Zur Problematik der Domestizierung der Löwen cf. P. Kuhlmann, Der Thron im Alten Ägypten, SDAIK 10, Glückstadt 1977, 87, der im Zusammenhang in Ägypten im Alten Reich das Motiv der Rosette ursprünglich nur für zahme Hoflöwen verwendet wurde. Diese Tiere habe man mit den Rosetten und Bändern aus Stoff oder Leder zu festlichen Anlässen geschmückt oder bemalt. Übernommen sei dieser Brauch aus Mesopotamien, wo die Rosette als Symbol der Ischtar galt.

Tatsächlich sehen die Haarwirbel bei den von Jequier angeführten frühen Beispielen wie applizierte Ornamente aus. Generell ist bei diesen Darstellungen neben dem Inhalt der zu erzielenden Aussage aber auch der Bildträger zu berücksichtigen, da tendenziell zu beobachten ist, daß die Haarwirbel in der Malerei technik- und materialbedingt eine größere Ähnlichkeit mit den natürlichen Haarbüscheln der Tiere aufweisen als die stilisierten Rosetten im Flachrelief".

mit den schreitenden Löwen neben dem Königsthron darauf hinweist, daß Zähmung und Haltung dieser Raubkatzen naturgegeben Schwierigkeiten mit sich brachten. Kuhlmann vermutet daher, daß es sich bei den Wiedergaben der Löwen am Königsthron nicht um lebendig vorgestellte Tiere, sondern vielmehr um rundplastische oder aufgemalte Bilder handelt.

Siehe dazu beispielsweise die Darstellung des Löwen an der Rampe am Tempel der Hatschepsut in Deir el- 


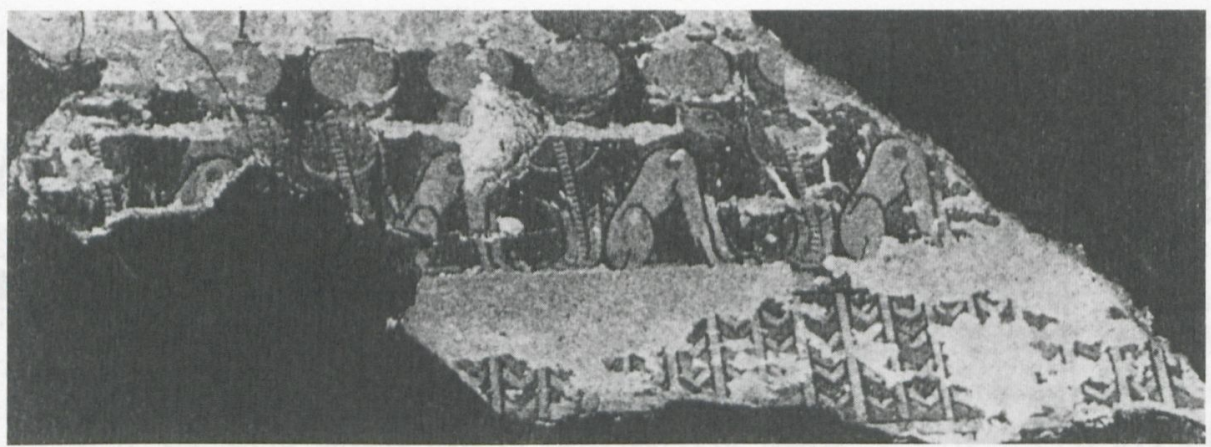

Abb. 8. Löwen im Baldachin-Fries eines Thrones im Grab des $H r-m-h b$ (TT 78)

In Ägypten sind stilisierte Formen der Wirbel, die der mesopotamischen Rosette vergleichbar oder von einem Kreis umschlossen sind, eher selten. Sie sind vorwiegend im Alten Reich zu finden, und im Fall der geschmückten Löwen am Thronsockel könnten archaisierende Tendenzen die Gestaltung der Wirbel als „Rosette“ hervorgerufen haben ${ }^{70}$.

Der Thron und der Thronkiosk als königliche Machtsymbole sind Bildträger für weitere Löwendarstellungen. Kuhlmann stellte in seiner Arbeit über die Throne verschiedene Motive der Löwen am Thron-

Bahari, bei dem der Schulterhaarwirbel von einem Kreis umgeben ist (s. die bibliographischen Angaben bei PM II ${ }^{2}$, 341). Arkell (in: JNES 7, 1948, 52) bemerkt zu dieser Ausführung der „Rosette“, daß der Künstler das Motiv von einem anderen kopiert haben könnte und selbst noch nie ein lebendiges Tier in der Natur gesehen haben mag, ihm also die Bedeutung dieses Details nicht bekannt war. Allerdings zeigt das Beispiel auf einem Turiner Papyrus, $\mathrm{daB}$ in der Malerei die Haarwirbel durchaus stilisiert ausgeführt sein können (s. die Abb. bei A. M. Donadoni u. a., Il Museo Egizio di Torino, Guida alla lettura di una civilità, Agostini 1988, 107). Umgekehrt zeigt die NR Kalksteinstele Moskau I.1.a. 5614 mit der Darstellung der Qadesch auf dem Löwen, daß auch im Relief der Künstler bemüht war, den Wirbeln eine naturnahe Form zu verleihen. Siehe dazu die Abb. bei S. Hodjash, O. Berlev, The Egyptian Reliefs and Stelae in the Pushkin Museum of Fine Arts, Moscow, Leningrad 1982, 74.

${ }^{70} \mathrm{Zu}$ einem der frühesten Beispiele für die Darstellung des Löwen mit "Schulterrosette" am Thron des Königs siehe G. Jequier, Les pyramides des reines Neit et Apouit, in: Fouilles à Saqqarah, Le Caire 1933, Taf. IV und V. In der Zeit nach dem Alten Reich wurde dieses Motiv unter Thutmosis III. und Schabaka kopiert. Cf. dazu J. Baines, Fecundity Figures, 89, sowie L. Habachi, Devotion of Tuthmosis III to his Predecessors: A Propos of a Meeting of Sesostris I with his Courtiers, in: Gs Mokhtar I, 349-359. sockel bzw. der Estrade und am Thronbaldachin vor $^{71}$. Ihr Vorhandensein wurde von ihm in erster Linie mit der Unterstreichung des königlichen Machtanspruches, der sich auch auf die Fremdländer erstreckt, begründet ${ }^{72}$.

Die Darstellung eines Löwenkopfes, der aus dem Lotoskapitell einer den Baldachin tragenden Säule herausragt, erwähnte Kuhlmann im Zusammenhang mit den Rinderprotomen, die oben am Abakus der Säule des Thronkiosks befestigt sein können ${ }^{73}$. Signifikant ist weiter die Friesdekoration eines Thronbaldachins mit alternierenden Uräusschlangen und Löwen, die jeweils die Sonnenscheibe auf dem Kopf tragen (Abb. 8) . $^{74}$. Die hockenden Löwen zeigen auch

${ }^{71}$ P. Kuhlmann, Der Thron im Alten Ägypten, ADAIK 10, Glückstadt 1977, 61 ff. und 85 ff.

${ }^{72}$ Kuhlmann, op. cit., 87, Anm. 7, weist den Löwen am Thron u. a. eine beschützende Funktion zu und sieht in ihnen weiterhin Abbilder des Königs, die diesen ,,in einem besonders furchterregenden Aspekt" darstellen. Siehe auch Pongracz, Löwendarstellungen an Podesten der Königsthrone, in: MDAIK 15, 1957, $213 \mathrm{ff}$., welche die unägyptischen „Schulterrosetten" sowie die über dem Löwenleib angegebenen Bänder als Hinweis auf die Beherrschung der Fremdländer interpretierte.

Siehe Kuhlmann, op. cit., Taf. 3 (Abb. 8) und p. 75 und 92. Der Löwenkopf ist nur schemenhaft zu erkennen. Cf. zum Motiv L. Borchardt, Die Aegyptische Pflanzensäule, Berlin 1897, 14 (Abb. 24) und 51 (Abb. 83). Nach Borchardt ist seit der Zeit Amenophis' II. diese Säulenform mit vier Löwenköpfen zwischen Kapitell und Abakus bezeugt.

${ }^{4}$ Siehe Kuhlmann, op. cit., Abb. 7 und cf. p. 91. Die Darstellung stammt aus der 18. Dyn. und befindet sich im Grab des Haremhab (TT 78). Nach der Publikation von A. und A. Brack, Das Grab des Haremheb, Theben Nr. 78, AV 35, Mainz 1980, p. 36 und Taf. 46 a und b, sind die Körper der Löwen gelb. Ihre Mähnen und die runden Flecken auf den Schultern wurden in blauer Farbe aufgemalt. 
hier einwandfrei den als runden Fleck auf der Schulter angegebenen Haarwirbel.

Kuhlmann klassifizierte die Löwen im Baldachinfries als apotropäischen Schutzdekor und wies ihnen die gleiche Funktion zu wie den Rinderprotomen am Abakus und den Löwen am Thronsockel. Einen eigenen Symbolgehalt des Thronbaldachins als Himmel hielt er für fraglich und deutete auch die Säulen, die das Dach des Thronkiosks tragen, rein funktional ${ }^{75}$.

Anders, als von Kuhlmann vermutet, erkenne ich in den Löwen eine Symbolik, die über die der Repräsentation des königlichen Machtanspruches, der die Fremdvölker einschließt, hinausgeht. Der Bildträger des Thrones an sich legt es zwar nahe, in der Anbringung der Tiere einen Hinweis auf den Machtanspruch und die Stärke Pharaos zu sehen, allerdings implizieren einige der Löwenmotive m. E. daneben eine Wiedergeburtssymbolik ${ }^{76}$. Denn wie sonst sollten sich die aus den Lotoskapitellen herauswachsenden Löwenköpfe und die im oberen Friesband die Sonne tragenden Löwen mit Schulterhaarwirbel erklären, die sicher von den Darstellungen der teils mit Bändern geschmückten Löwen am Sockel bzw. am Podest und der Armlehne zu differenzieren sind?

Ich möchte zwischen dem irdischen Machtanspruch und der Regenerationsthematik unterscheiden, da das, was im Großen für die Tempeldekoration gilt, sich im Kleinen im Hinblick auf die Darstellung der Löwen auch im Dekorationsprogramm des Thronkioskes feststellen läßt $\mathrm{t}^{77}$. Unten am Thron drückt sich der irdische Machtanspruch des Herrschers in den schreitenden oder mit Bändern geschmückten und damit bezwungenen Löwen am Sockel und Podest aus, während oben in Himmelsnähe die Löwen mit der Sonnenscheibe auf dem Kopf sowie der Löwenkopf, der aus der Lotossäule

Kuhlmann, op. cit., $91 \mathrm{f}$.

${ }^{6}$ Zur Lotossäule als Symbol der Wiedergeburt beim Sonnenaufgang cf. D. Arnold, in: LÄ V, 345, s. v. „Säule". Zum Löwen und dem Lotos als Regenerationssymbol cf. auch Hornung, Die Tragweite der Bilder, 227, Abb. 20 (= Sarg Alexandria 32). Cf. ferner die Vignette zu $\mathrm{Tb}$ 15, die ein Löwenpaar vor dem Lotosdickicht sitzend zeigt.

Zu den allgemeinen Prinzipien der Tempeldekoration siehe D. Kurth, Eine Welt aus Stein, Bild und Wort - Gedanken zur spätägyptischen Tempeldekoration, in: J. Assmann und G. Burkard (Hrsg.), 5000 Jahre Ägypten - Genese und Permanenz pharaonischer Kunst, Heidelberg 1983, 89 ff.; J. Assmann, Das kulturelle Gedächtnis, München 1992, $177 \mathrm{ff}$. herausragt, angebracht sind. Im Zentrum dieses Mikroskosmos' oder Weltgebäudes thront Pharao, die chaotische fremde Welt und die wilde Natur unter seinen Sandalen, gestärkt und geschützt durch das Löwendekor am Thronsitz und sich über die Löwendarstellungen im Fries und am Kapitell der zyklisch wiederkehrenden Regeneration gewi ${ }^{78}$.

\section{§3 Der Haarwirbel im Schulterfell der Totenfresserin}

Die „Große Fresserin“ der Totengerichtsszene des Totenbuchspruches 125 stellt ein Mischwesen dar, dessen Leib sich aus verschiedenen Tierkörperteilen zusammensetzt. Der Kopf der Totenfresserin mit der länglichen, oft aufgerissenen Schnauze, in der die spitzen Zähne zu sehen sind, besteht aus einem Krokodilskopf, das gedrungene Hinterteil aus dem eines Nilpferds und der Rumpf aus dem eines Löwen ${ }^{79}$.

Kommt dem fürchterlichen Höllenwesen die Funktion zu, die gescheiterten Kandidaten des Gerichts zu verschlingen und damit zu ewiger Verdammnis zu verurteilen, setzt das natürlich entsprechende physische Kräfte voraus. Ein kraftvolles, furchterregendes Tier übt auf den Betrachter nicht nur die erzielte beängstigende Wirkung aus, sondern kann die gestellte Aufgabe zweifellos besser erfüllen als ein schwaches Tier. Daher liegt es nahe, in den Darstellungen der Totenfresserin mehrmals die

${ }^{78} \mathrm{Cf}$. in diesem Zusammenhang die von De Wit, Le rôle et le sens du lion, $158 \mathrm{f}$., vertretene These, dass der Löwenthron ein Abbild der Welt darstellt. Anders Kuhlmann, op. cit., 86 .

79 Siehe zur „Großen Fresserin“ Seeber, Totengericht, $163 \mathrm{ff}$. Als Belege für die „Rosette“ am Schulterfell der Totenfresserin führt Seeber an: pBM 10470; pBerlin P 3002; pLouvre 3068; pKairo G 4885; pLeiden T 4; TT 336; pBM 9932 (Seeber, op. cit., 163, Anm. 747). In den die Ikonographie der Totenfresserin beschreibenden Beischriften heißt es: „Ihr Vorderteil ist das eines Krokodils, ihr Hinterteil ein Nilpferd und ihre Mitte ein Löwe“ (s. die Vignette zu Tb 125 im pHunefer). Cf. ferner z. B. die Darstellungen der Totenfresserin mit Haarwirbel im pGreenfield (s. E. A. W. Budge, The Greenfield Papyrus in the British Museum, The Funerary Papyrus of Princess Nesitanebtashru, Daughter of Painetchem II and Nesi-Khensu, and Priestess of Amen-Ra at Thebes, about B.C. 970, London 1912, Taf. XCIV). Für die Grabdekoration s. beispielsweise E. Feucht, Das Grab des Nefersecheru (TT 296), Theben 2, Mainz 1985, Farbtafel Ib und Taf. X. 


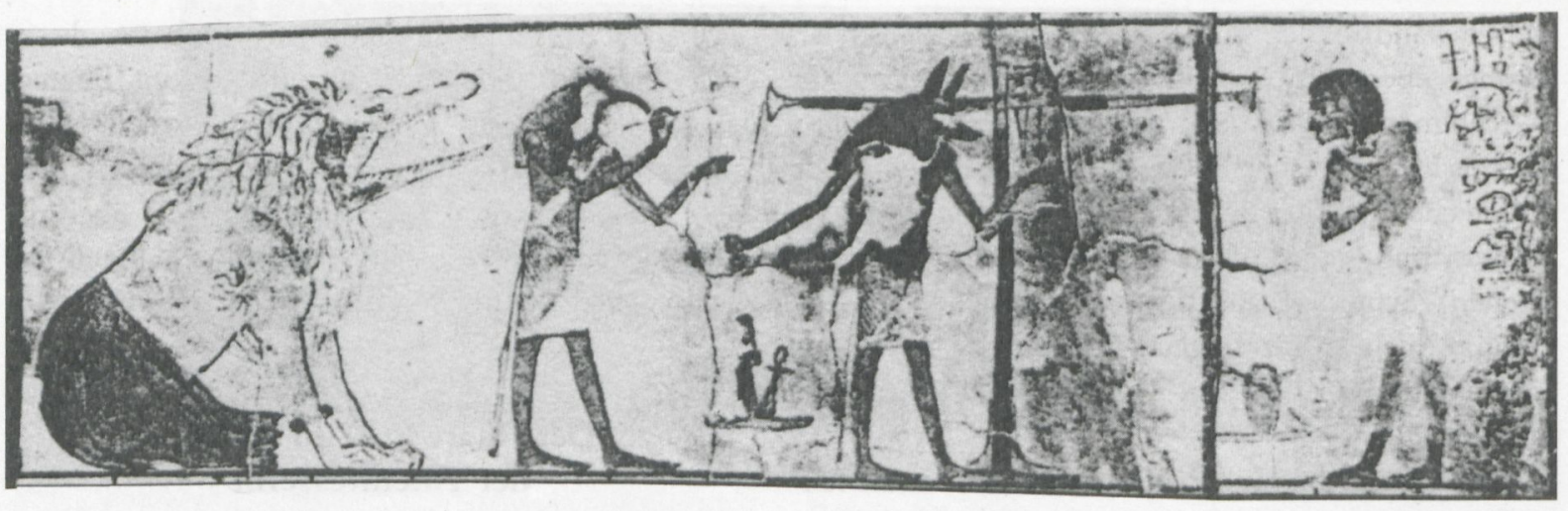

Abb. 9. Totenfresserin im pBM 10473

Haarwirbel als Kennzeichen der körperlichen Stärke der Bestie wiederzufinden. Als Beispiele mögen die Darstellungen der Totenfresserin im pBM 10473 (Abb. 9), im pBM 10470 (Tafel XX) und im Grab TT 336 (Abb. 10) genügen ${ }^{80}$.

\section{\$4 Die Göttin Qadesch auf dem Löwen}

Mehrere Stelen zeigen die Göttin Qadesch als eine unbekleidete Frau mit Sonnenscheibe und Mondsichel auf der Hathorperücke sowie mit Lotosblüten und einer oder mehreren Schlangen in den Händen. Die in Syrien/Palästina beheimatete Göttin steht dabei in Frontansicht auf einem in Schrittstellung abgebildeten Löwen. Bei einigen Dokumenten ist die Schulter des Löwen deutlich mit dem Schulterhaarwirbel verziert. Ein schönes Beispiel stellt die Abbildung der Göttin auf der Stele Turin 50066 dar (Tafel XXI) ${ }^{81}$. Der schreitende Löwe, mit imposanter Mähne, schwarz verziertem Auge und geflecktem Fell, zeigt auf seiner Schulter den Wirbel, der allerdings leicht verrutscht ist und nicht direkt auf dem Schultergelenk sitzt.

${ }^{80}$ Abb. 9 nach S. R. K. Glanville, Note on the Nature and the Date of the Papyri of Nakht, BM 10471 ND 10473, in: JEA 13, 1927, Taf. 21 b (pBM 10473; 19. Dyn.); Tafel XX nach E. Dondelinger, Papyrus Ani, Codices Selecti LXII, Graz 1978, Taf. 3; Abb. 10 nach B. Bruyère, Fouilles de Deir el Médineh (1924-25), FIFAO 3, 3, Le Caire 1926, 83, Fig. 54.

${ }^{81}$ Siehe die Abb. und die bibliographischen Angaben bei I. Cornelius, The Iconography of the Canaanite Gods Reshef and Bacal, OBO 140, Freiburg (Schweiz) 1994, Taf. 20 (RR 28) und 57-60. Die Stele stammt aus Deir el-Medineh und ist in die Zeit Ramses' II. datiert.
Auch für diese Beispiele der Angaben der Haarwirbel lassen sich mehrere Erklärungen finden. Der die Qadesch tragende Löwe mit dem Haarwirbel in seinem Fell könnte zum einen den Aspekt der Fruchtbarkeit der Göttin unterstreichen. Auf einer weiteren Bedeutungsebene ist dieses Bild $\mathrm{m}$. E. wie die Darstellung der Horizontlöwen oder des Thronensembles als Wiedergabe eines Weltbildes zu „lesen“.

Auf ihrem Kopf trägt die Göttin die Gestirne in Form von Sonnenscheibe und Mondsichel, was ihre Beziehung zum Himmel verdeutlicht. Als Herrin der Tiere und des irdischen Bereiches hat sie den Löwen unter sich. Als Herrin der Fruchtbarkeit und des Lebens steht sie im Zentrum der Bildkomposition und hält in ihren Händen Lotosblumen und Schlan-

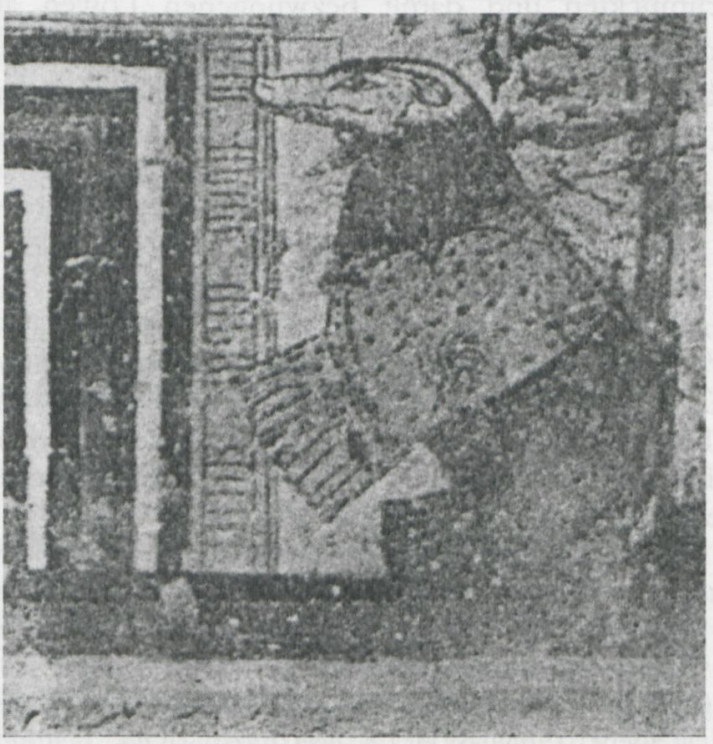

Abb. 10. Totenfresserin im Grab TT 336 

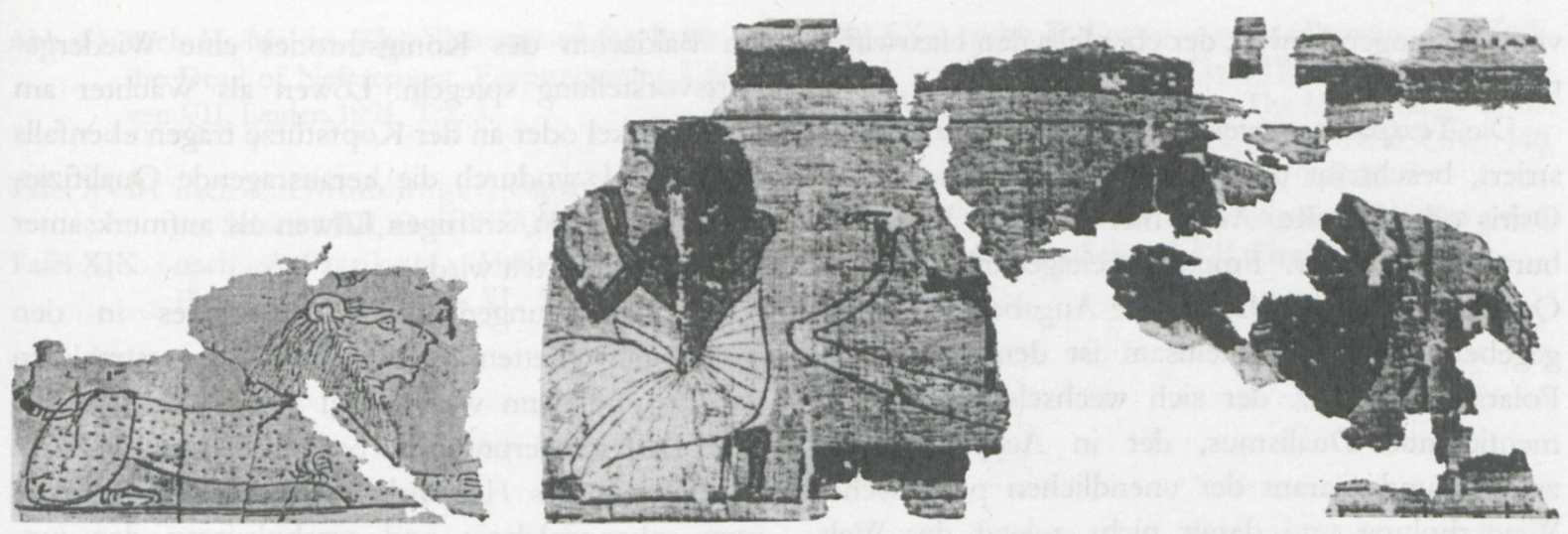

Abb. 11. Totenbuch des Nfr-rnpt, Vignette zu Spruch 17

gen, Attribute, die ich als Symbole für Leben und Tod interpretiere ${ }^{82}$.

In den Dreiergruppen, die Qadesch mit Amun-Min und Reschep bildet, wird diese Vermutung m. E. bestätigt, denn in diesen Darstellungen hält die Göttin regelhaft den Lotos an die Nase des einheimischen Fruchtbarkeitsgottes Amun-Min und die Schlangen an die des fremdländischen, kriegerischen Gottes Reschep, der seine todbringenden Waffen, das Sichelschwert und die Lanze, in den Händen hält ${ }^{83}$.

${ }^{82}$ Anders O. Keel, Das Recht der Bilder gesehen zu werden, OBO 122, Freiburg/Schweiz 1992, 203-208 und spez. 208. Nach Keel drückt die Darstellung die Herrschaft der Qadesch über die sexuelle Potenz und Lebenskraft aus (ähnliche Deutung bei Stadelmann, in: LÄ V, 26, s. v. „Qadesch"). Die Schlange, welche die Göttin in der Hand hält und an die Nase des Reschep führt, bringt Keel mit der Heilkraft, die sowohl Qadesch als auch Reschep anhaftet, in Verbindung. Die Schlange symbolisiert nach seiner Interpretation wie der Lotos die Lebendigkeit, Erotik und Lebenskraft.

${ }^{83}$ Bei einer anderen Darstellung der Göttin auf einer Stele des Neuen Reiches, die sich heute in Moskau befindet, trägt der Löwe, der sein Maul drohend aufgerissen hat, um seinen Leib die erstmals bei Pepi II. bezeugten Bänder und den Schulterhaarwirbel (siehe S. Hodjash, O. Berlev, The Egyptian Reliefs and Stelae in the Pushkin Museum of Fine Arts, Moscow, Leningrad 1982, 74 und cf. zu den Bändern oben Anm. 9 und 72). Vor ihm stehen der Stelenstifter und seine Frau. Vielleicht war die Anwesenheit dieser beiden Privatpersonen ausschlaggebend dafür, dem Löwen in diesem Kontext die Schmuck(?)-Bänder zu verleihen und ihn dadurch als bezwungenes Tier (cf. dazu die Theorie von Jequier, s. o. Anm. 68) zu kennzeichnen, das dem Adoranten nicht gefährlich werden konnte. Andererseits können die Bänder Ausdruck der fremdländischen Herkunft der Göttin sein, wie es Pongracz (in: MDAIK 15, 1957, 216ff.) herausgearbeitet hat, oder in

\section{Schlußbetrachtung}

Am Ende der hier angestellten Betrachtungen zur Bedeutung der Wirbel im Schulterfell der Löwen komme ich auf weitere Löwendarstellungen in Vignetten des Totenbuchkapitels 17 zurück, die ebenfalls das ikonographische Element des Haarwirbels enthalten und die bislang konstatierten Funktionen, die mit der Angabe verbunden sind, bestätigen.

Die erste Szene zeigt einen Löwen auf einem Podest liegend (Tafel XXII) ${ }^{84}$. Über dem Löwen ringelt sich eine Kobra im Pflanzendickicht, neben dem außerdem ein Flammenzeichen zu sehen ist. Der dazugehörige Text weist die Uräusschlange als Wadjet aus, als die Herrin des verzehrenden Feuers, die als Auge des Re die Feinde vernichtet ${ }^{85}$. Vor dem Löwen sieht man wieder einen Opferaltar mit Lotosblüte, die diesmal zur Barke hin zeigt, in der der gealterte Sonnengott Atum in einer Scheibe zu sehen ist. Auch hier macht es Sinn, daß der Löwe als Kennzeichen seiner Jugend in Opposition zum gealterten Sonnengott Atum den Haarwirbel an der Schulter trägt.

Die Vignette zu diesem Spruch im Totenbuch des Nfr-rnpt zeigt als Variante statt des Altars mit der Lotosblüte den Verstorbenen im Anbetungsgestus

einer Tradition stehen, in der archaisierende Elemente zitiert wurden.

${ }^{84}$ pBM 10470 s. E. Dondelinger, Papyrus Ani, Codices Selecti LXII, Graz 1978, Taf. 10.

${ }^{85}$ Siehe Urk. V, 88,5. Zum gemeinsamen Auftreten von Löwe und Uräusschlange cf. oben die Darstellungen am Thronkiosk und die Darstellung im Grab TT 2 (= Abb. 5) sowie Westendorf, Uräus und Sonnenscheibe, in: SAK 6, 1978, $201 \mathrm{ff}$. 
vor dem jungen Löwen, der ebenfalls den Haarwirbel trägt (Abb. 11) ${ }^{86}$.

Die Textpassage, welche diese Darstellungen illustriert, beschreibt den Löwen als den Phallus des Osiris oder des Re. Auch hier mag der Wiedergeburtsgedanke bzw. Fruchtbarkeitsgedanke wie bei Qadesch den Ausschlag für die Angabe des Wirbels gegeben haben ${ }^{87}$. Gemeinsam ist den Bildern die Polarisierung bzw. der sich wechselseitig komplementierende Dualismus, der in Ägypten Kennzeichen und Garant der unendlichen periodischen Wiederholung und damit nicht zuletzt der Weltschöpfung ist. Dem gealterten Sonnengott Atum in seiner Barke steht der durch den Schulterhaarwirbel gekennzeichnete junge Sonnengott in Löwengestalt gegenüber. Zwischen beiden hockt der Verstorbene, den Blick auf den verjüngten Gott gerichtet.

Die angeführten Beispiele zeigen in ihrer Breite und Schlüssigkeit, daß die Angabe des Haarwirbels bei Löwendarstellungen keineswegs nur zufällig ist, sondern vielmehr wichtiger Bestandteil des Motivs sein kann, der dem Betrachter einen Inhalt signalisieren soll, dessen Bedeutung von ihm vielleicht nicht mit dem ersten, sondern erst mit dem zweiten Blick erschlossen und erfaßt werden kann.

Insofern können die erstmals von Garnot und später von Arkell und Bate ausgeführten Beobachtungen bestätigt werden. Auf Ägypten bezogen läßt sich festhalten, daß die Haarwirbel im Schulterfell der Löwen das Tier als junges, kräftiges Tier qualifizieren. Ihre Existenz am Objekt mag neben dem Kontext und dem zu vermittelnden Inhalt von der Qualität des Kunstwerkes bzw. dem Ausbildungsstand seines Künstlers abhängen.

Erscheint der mit dem Haarwirbel versehene Löwe in Verbindung mit dem König, lassen sich Bezüge zur physischen Stärke des Herrschers und seiner Regeneration herstellen. Pharao ist wie ein Löwe in seiner Kraft und mißt sich während des Höhepunktes der Löwenjagd mit dem stärksten Tier des Rudels. Der neben dem Thron schreitende Löwe signalisiert die Vitalität des Königs, während die Löwen

${ }^{86}$ Siehe M. Milde, The Vignettes of the Book of the Dead of Neferrenpet, Egyptologische Uitgaven VII, Leiden 1991, Taf. 3.

${ }^{7}$ U. Rößler-Köhler, Kapitel 17 des Ägyptischen Totenbuches, GOF 10, Wiesbaden 1979, 218 (n. 2) und 235 , bringt ihn allerdings mit einer ,kämpferischen Rolle des Phallus " in Verbindung und verweist dazu auf den $R w$ Löwen als Gottesphallus. am Baldachin des Königsthrones eine Wiedergeburtsvorstellung spiegeln. Löwen als Wächter am Thronsockel oder an der Kopfstütze tragen ebenfalls den Wirbel, wodurch die herausragende Qualifizierung des jungen, kräftigen Löwen als aufmerksamer Wächter vermittelt wird.

In Darstellungen des Löwenpaares in den Totenbuchvignetten zu Spruch 17 ist erstmals in der 19. und dann vorwiegend in der 21. Dynastie eine Differenzierung der beiden Löwen feststellbar. Mittels des Haarwirbels werden die Tiere als jung gekennzeichnet und symbolisieren den jungen, regenerierten gegenüber dem gealterten Sonnengott.

\section{SUMMARY}

Until now in ancient Egyptian representations, the shoulder ornament as found on lions, has been explained as a decorative element only. Based on the observation of nature that cowlicks occur in the shoulder fur of young lions, the author demonstrates that this iconographic detail adds a symbolic meaning to the image. The example of the vignette of $\mathrm{BD}$ Spell 17 with the two lions of the horizon proves that by indicating such cowlicks, the young animal, i.e. the regenerated and youthful sun god, is distinguished from the older one.

\section{Abbildungsnachweis}

Abb. 1 nach J. Vandier, La Tombe de Nefer Abou, MIFAO 69, Le Caire 1935, Frontispice.

Abb. 2 nach E. A. W. Budge, The Greenfield Papyrus in the British Museum, The Funerary Papyrus of Princess Nesitanebtashru, Daughter of Painetchem II and Nesi-Khensu, and Priestess of Amen-Ra at Thebes, about B.C. 970, London 1912, Taf. L.

Abb. 3 nach B. Bruyère, Tombes thébaines de Deir el Médineh à décoration monochrome, MIFAO 86, Le Caire 1952, Taf. 3.

Abb. 4 nach E. Chassinat, La seconde trouvaille de Deir el-Bahari (sarcophages), Leipzig 1909, p. 26, fig. 24.

Abb. 5 nach $\mathrm{Tb}$ (Naville), Taf. 30, A. g.

Abb. 6 nach W. Kaiser (Hrsg.), Ägyptisches Museum Berlin, Berlin 1967, Nr. 720.

Abb. 7 nach G. Foucart, Tombes thébaines, Le tombeau d'Amonmos, MIFAO 57, Le Caire 1932, Taf. 28.

Abb. 8 nach P. Kuhlmann, Der Thron im Alten Ägypten, ADAIK 10, Glückstadt 1977, Abb. 7.

Abb. 9 nach S. R. K. Glanville, Note on the Nature and the Date of the Papyri of Nakht, BM 10471 ND 10473, in: JEA 13, 1927, Taf. 21 b.

Abb. 10 nach B. Bruyère, Fouilles de Deir el Médineh (1924-25), FIFAO 3,3, Le Caire 1926, p. 83, fig. 54. 
Abb. 11 nach H. Milde, The Vignettes of the Book of the Dead of Neferrenpet, Egyptologische Uitgaven VII, Leiden 1991, Taf. 3.

Tafel XVIII nach E. Dondelinger, Papyrus Ani, Codices Selecti LXII, Graz 1978, Taf. 7.

Tafel XIX nach A. Piankoff, Mythological Papyri, Plates, Bollingen Series XL. 3, Taf. 1.
Tafel XX

nach E. Dondelinger, Papyrus Ani, Codices Selecti LXII, Graz 1978, Taf. 3.

Tafel XXI nach I. Cornelius, The Iconography of the Canaanite Gods Reshef and Bacal, OBO 140, Freiburg (Schweiz) 1994, Taf. 20.

Tafel XXII nach E. Dondelinger, Papyrus Ani, Codices Selecti LXII, Graz 1978, Taf. 10. 


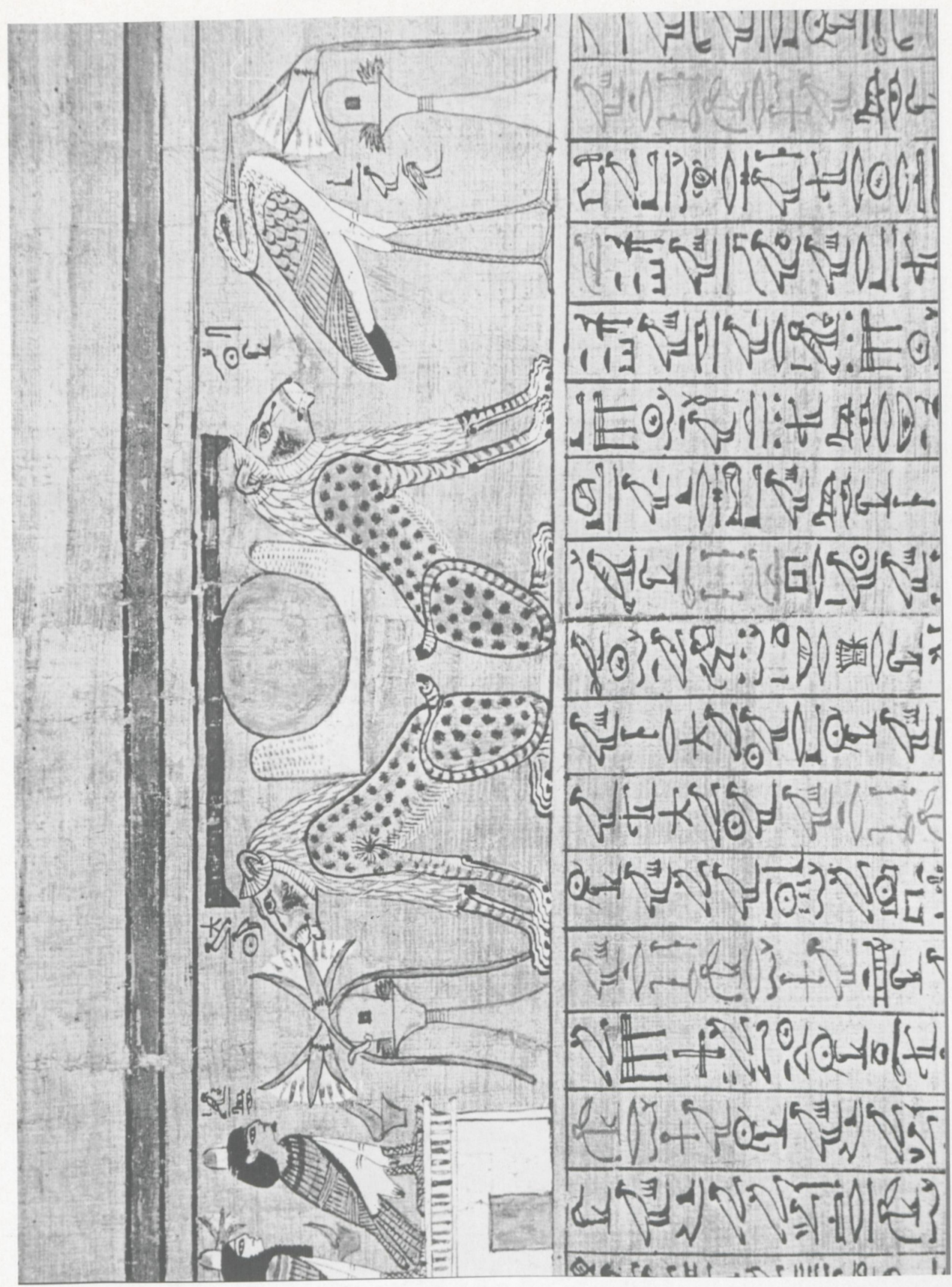




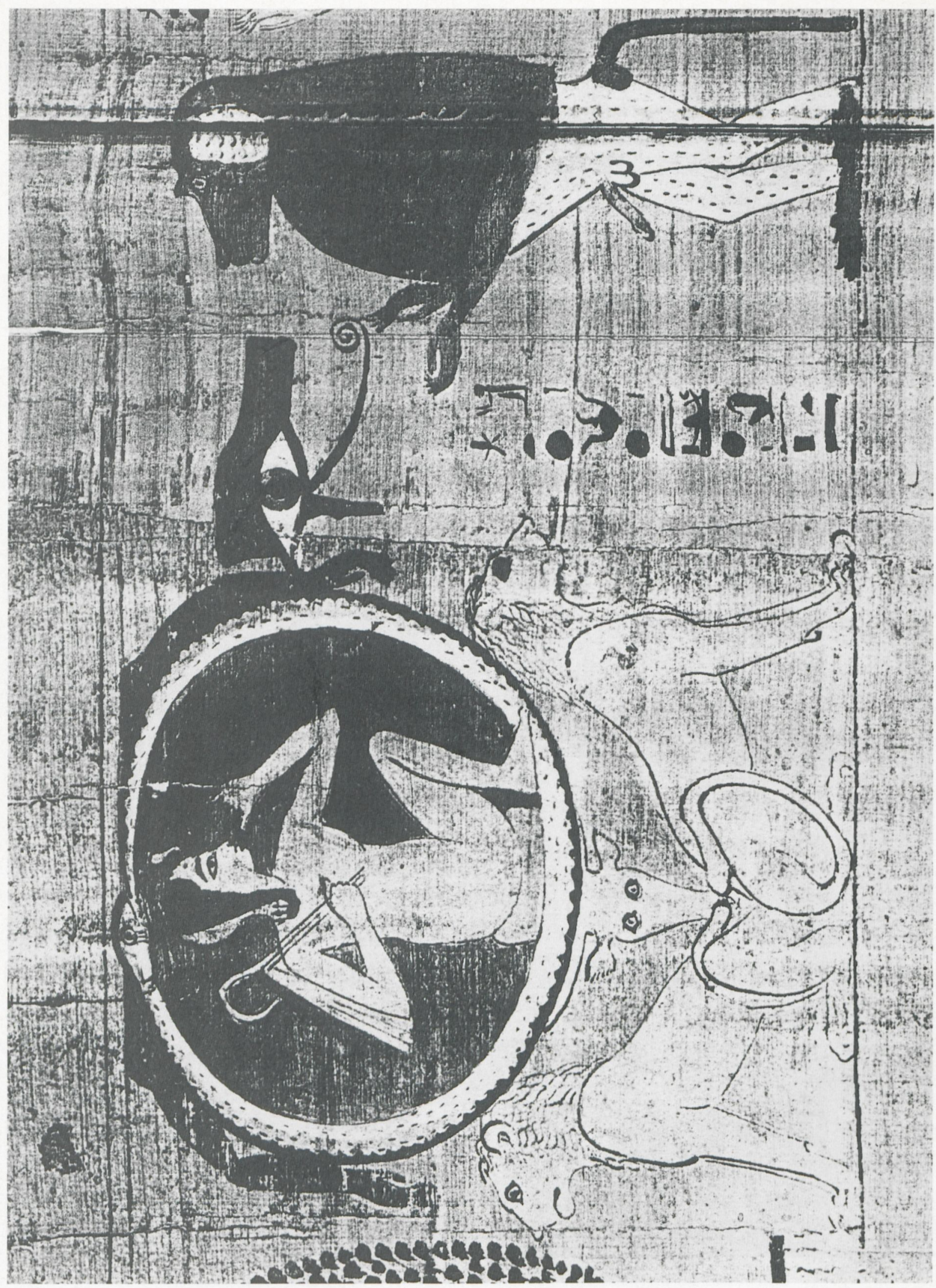

Der junge Sonnengott im Uroboros, Mythologischer Papyrus der Hrjt-wbn (zu Budde, Schulterrosette) 
| MA $=4+4=4 \mid$

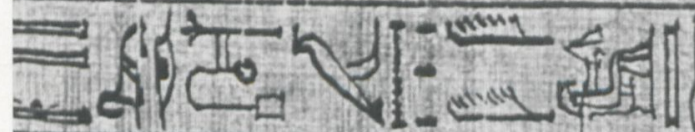

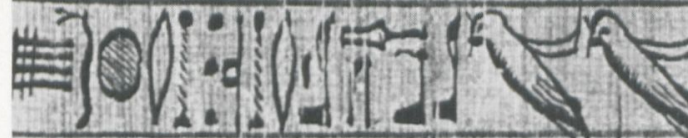

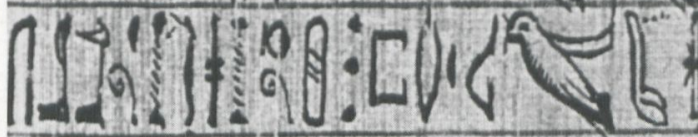

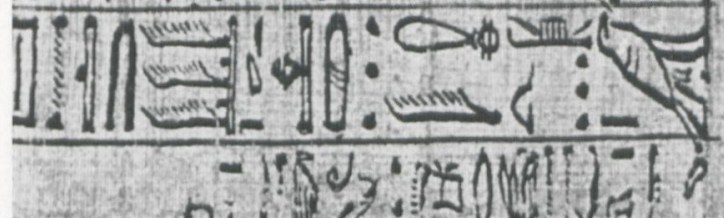
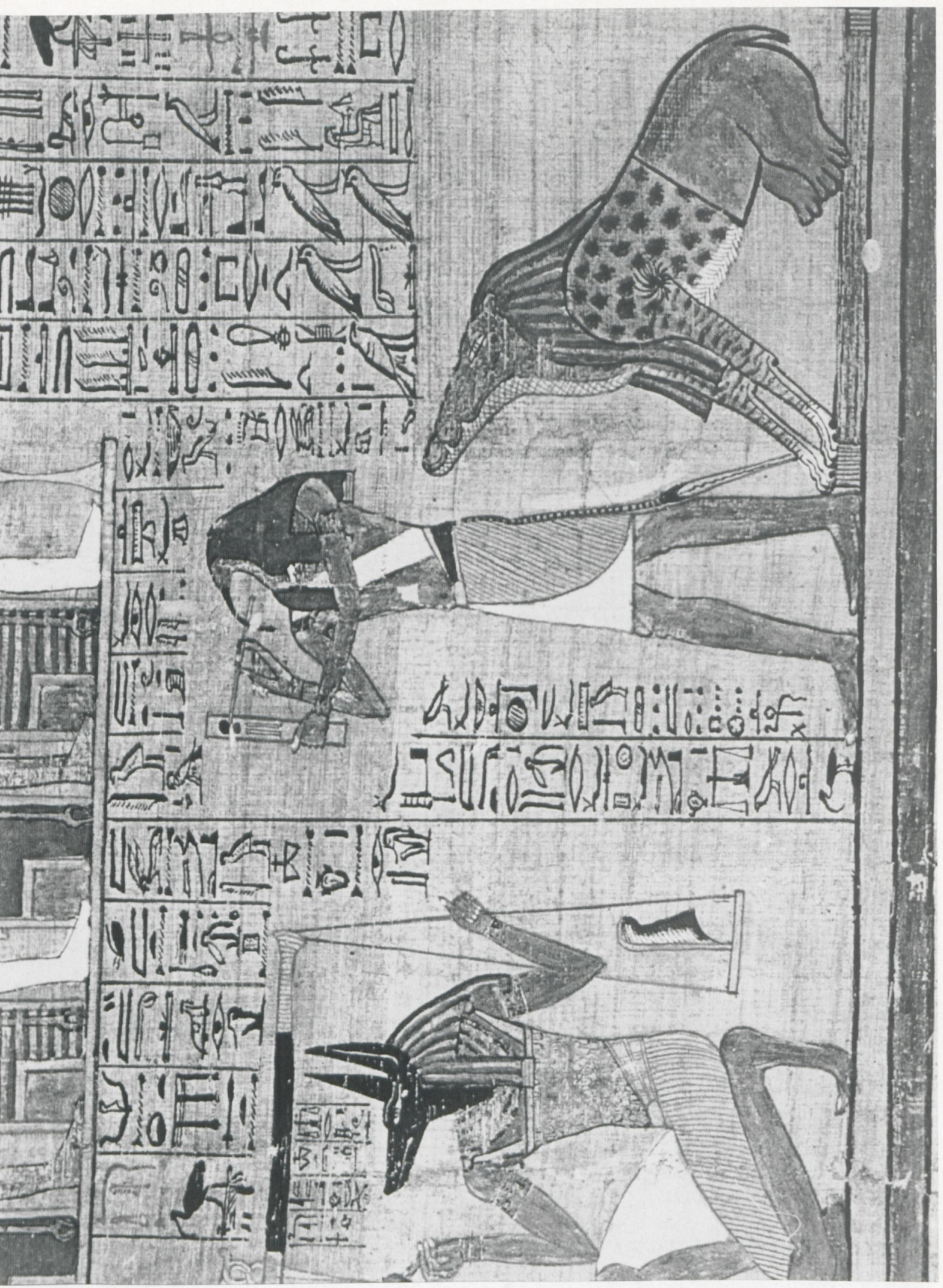

Totenfresserin im pBM 10470 (zu Budde, Schulterrosette) 


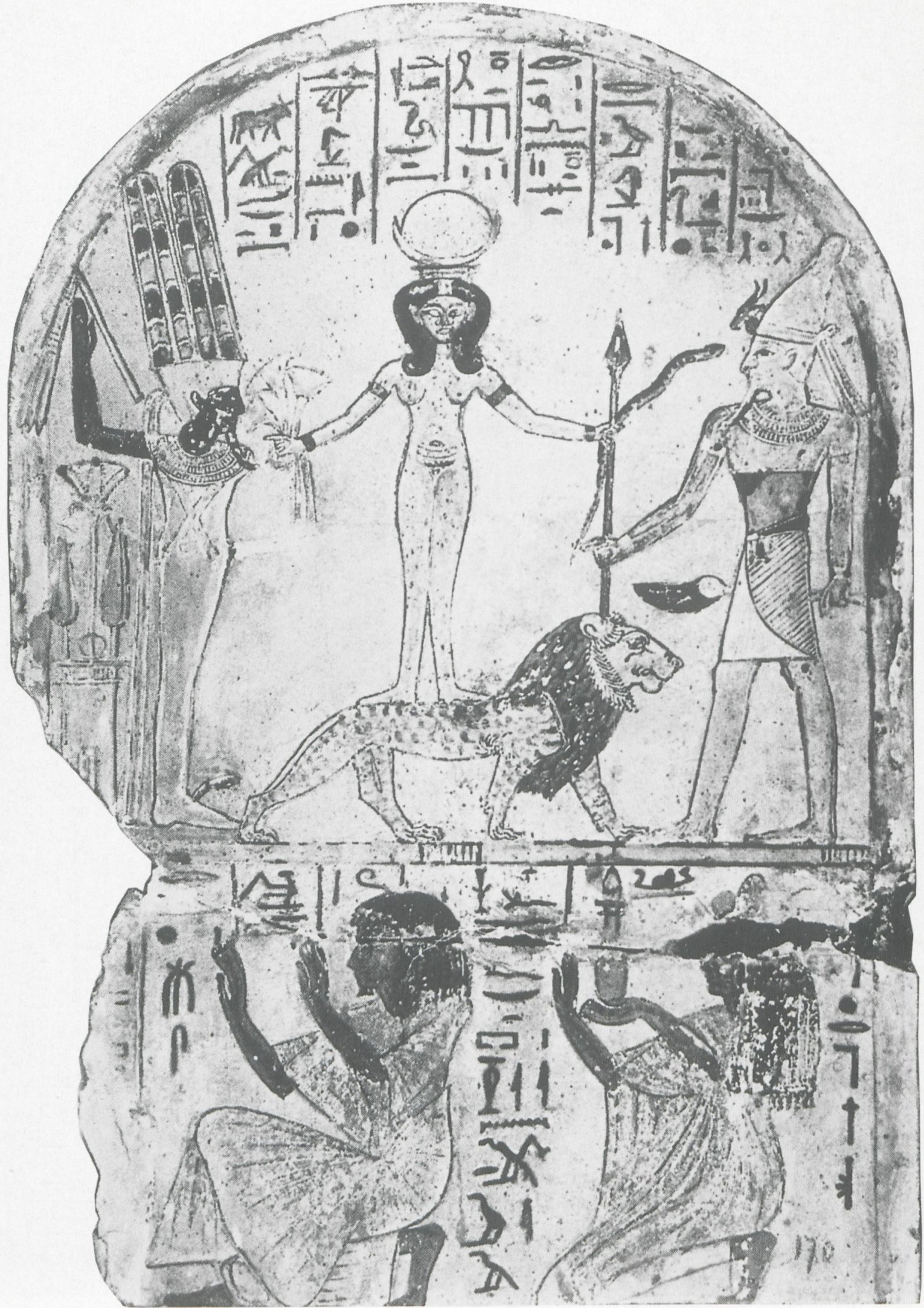

Qadesch auf dem Löwen (Stele Turin 50066) (zu Budde, Schulterrosette) 


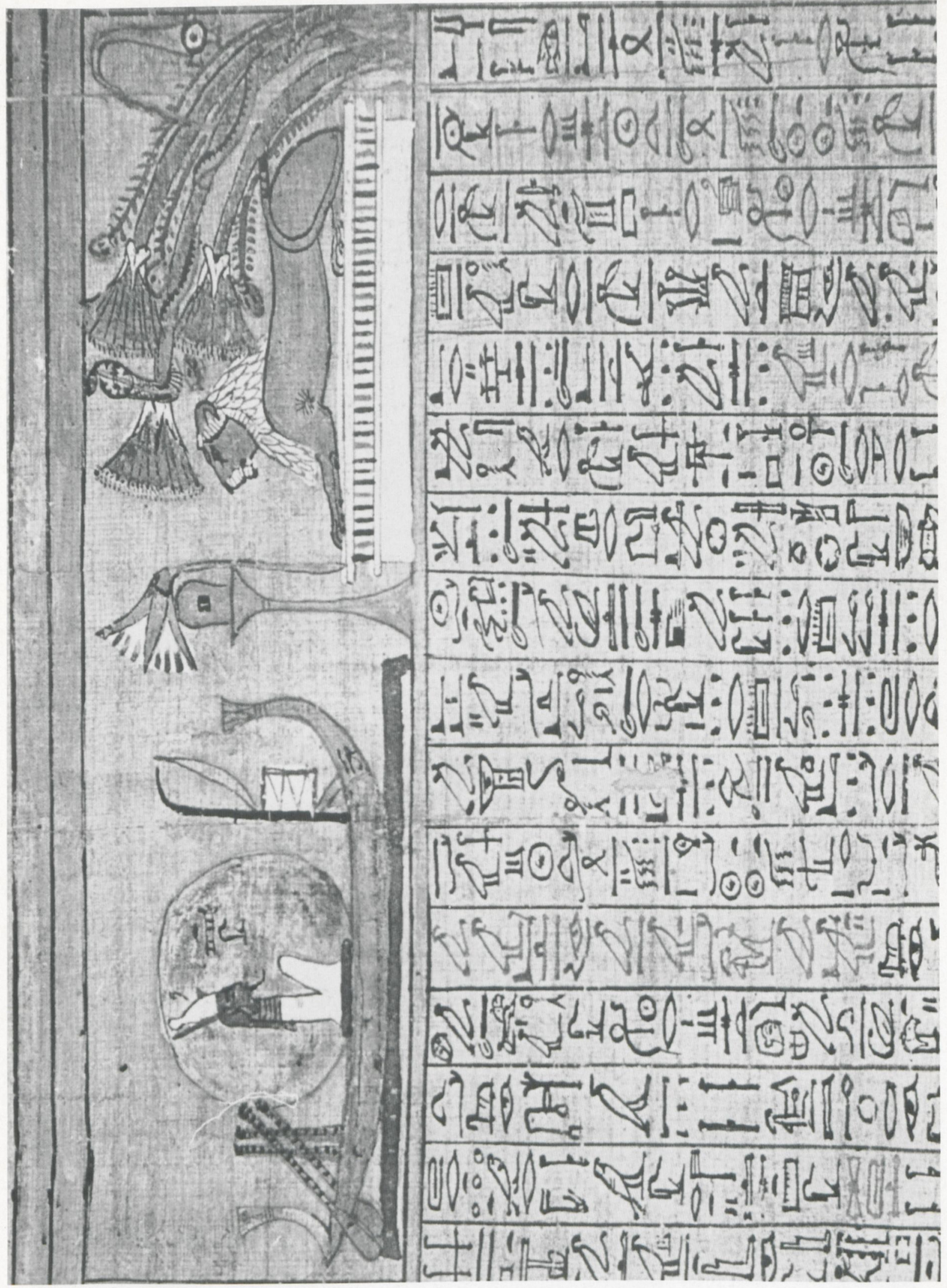

Totenbuch des 3nj (pBM 10470), Vignette zu Spruch 17 (zu Budde, Schulterrosette) 